\title{
Left-right symmetry and the charged Higgs bosons at the LHC
}

\section{G. Bambhaniya, ${ }^{a}$ J. Chakrabortty, ${ }^{b}$ J. Gluza, ${ }^{c}$ M. Kordiaczyńska ${ }^{c}$ and R. Szafron ${ }^{d}$}

a Theoretical Physics Division, Physical Research Laboratory,

Navarangpura, Ahmedabad - 380009, India

${ }^{b}$ Department of Physics, Indian Institute of Technology,

Kanpur - 208016, India

${ }^{c}$ Institute of Physics, University of Silesia,

Uniwersytecka 4, PL-40-007 Katowice, Poland

${ }^{d}$ Department of Physics, University of Alberta, Edmonton, AB T6G 2E1, Canada

E-mail: gulab@prl.res.in, joydeep@iitk.ac.in, gluza@us.edu.pl, mkordiaczynska@us.edu.pl, szafron@ualberta.ca 
ABSTRACT: The charged Higgs boson sector of the Minimal Manifest Left-Right Symmetric model (MLRSM) is investigated in the context of LHC discovery search for new physics beyond Standard Model. We discuss and summarise the main processes within MLRSM where heavy charged Higgs bosons can be produced at the LHC. We explore the scenarios where the amplified signals due to relatively light charged scalars dominate against heavy neutral $Z_{2}$ and charged gauge $W_{2}$ as well as heavy neutral Higgs bosons signals which are dumped due to large vacuum expectation value $v_{R}$ of the right-handed scalar triplet. Consistency with FCNC effects implies masses of two neutral Higgs bosons $A_{1}^{0}, H_{1}^{0}$ to be at least of $10 \mathrm{TeV}$ order, which in turn implies that in MLRSM only three of four charged Higgs bosons, namely $H_{1,2}^{ \pm \pm}$and $H_{1}^{ \pm}$, can be simultaneously light. In particular, production processes with one and two doubly charged Higgs bosons are considered. We further incorporate the decays of those scalars leading to multi lepton signals at the LHC. Branching ratios for heavy neutrino $N_{R}, W_{2}$ and $Z_{2}$ decay into charged Higgs bosons are calculated. These effects are substantial enough and cannot be neglected. The tri- and four-lepton final states for different benchmark points are analysed. Kinematic cuts are chosen in order to strength the leptonic signals and decrease the Standard Model (SM) background. The results are presented using di-lepton invariant mass and lepton-lepton separation distributions for the same sign (SSDL) and opposite sign (OSDL) di-leptons as well as the charge asymmetry are also discussed. We have found that for considered MLRSM processes tri-lepton and four-lepton signals are most important for their detection when compared to the SM background. Both of the signals can be detected at $14 \mathrm{TeV}$ collisions at the LHC with integrated luminosity at the level of $300 \mathrm{fb}^{-1}$ with doubly charged Higgs bosons up to approximately $600 \mathrm{GeV}$. Finally, possible extra contribution of the charged MLRSM scalar particles to the measured Higgs to di-photon $\left(H_{0}^{0} \rightarrow \gamma \gamma\right)$ decay is computed and pointed out.

KeYwords: Beyond Standard Model, Higgs Physics, GUT, Standard Model 


\section{Contents}

1 Introduction 1

2 MLRSM processes with charged Higgs boson particles at the LHC 3

2.1 Primary production of heavy charged Higgs bosons at the LHC 9

2.1.1 $\quad p p \rightarrow H_{1}^{+} H_{1}^{-}$and $p p \rightarrow H_{2}^{+} H_{2}^{-} \quad 9$

2.1.2 $p p \rightarrow H_{1}^{++} H_{1}^{--}$and $p p \rightarrow H_{2}^{++} H_{2}^{--} \quad 10$

2.1.3 $p p \rightarrow H_{1}^{ \pm \pm} H_{1}^{\mp}$ and $p p \rightarrow H_{2}^{ \pm \pm} H_{2}^{\mp} \quad 12$

$\begin{array}{lll}2.2 & \text { Primary production of a heavy Higgs and gauge bosons } & 14\end{array}$

2.2.1 $p p \rightarrow W_{1 / 2}^{\mp} H_{1 / 2}^{ \pm}, p p \rightarrow Z_{1 / 2} H_{1 / 2}^{ \pm}$and $p p \rightarrow \gamma H_{1 / 2}^{ \pm} \quad 14$

3 Simulations and results for final lepton signals $\quad 14$

$\begin{array}{lll}3.1 & \text { Events selection criteria } & 14\end{array}$

3.2 Signal events for doubly charged Higgs particles in MLRSM 15

3.2.1 $p p \rightarrow H_{1}^{++} H_{1}^{--}$and $p p \rightarrow H_{2}^{++} H_{2}^{--} \quad 16$

$\begin{array}{lll}3.2 .2 & p p \rightarrow H_{1}^{ \pm \pm} H_{1}^{\mp} \text { and } p p \rightarrow H_{2}^{ \pm \pm} H_{2}^{\mp} & 19\end{array}$

$\begin{array}{lll}3.3 & \text { Background estimation and significance of signals } & 20\end{array}$

4 MLRSM charged Higgs bosons contribution to $H_{0}^{0} \rightarrow \gamma \gamma \quad 21$

5 Conclusions and outlook $\quad 24$

A Reconciling FCNC effects and large $v_{R}$ with relatively light charged Higgs $\begin{array}{ll}\text { mass spectrum within MLRSM } & \mathbf{2 6}\end{array}$

\section{Introduction}

The LHC machine is working incredibly well shifting up the discovery limits for all the non-standard masses. For the same reason it is also true for the non-standard couplings and their possible values are shrinking more and more. Good examples are parameters connected with Left-Right (LR) symmetric models. These models enjoy richness of several types of beyond-the-SM particles [1,2]. No wonder that these models are interesting for theoretical and phenomenological studies, for some recent works see [3-9] and explored also by the LHC collaborations.

The searches at CMS and ATLAS have tightened up the limits on the masses of heavy gauge bosons. Let us mention that before the LHC era the fits to low energy charged and neutral currents were quite modest, e.g. for a charged gauge boson PDG reports $M_{W_{2}}>715 \mathrm{GeV}[10,11]$. The new LHC analysis pushed the limits already much above $2 \mathrm{TeV}$ [12-18]. All these searches provide robust bounds on the extra gauge bosons, for 
instance, the present limit for a charged heavy boson coming from the "golden" decay chain $W_{R} \rightarrow l_{1} N_{l} \rightarrow l_{1} l_{2} j j$ is $[17,18]$

$$
M_{W_{2}} \geq 2.8 \mathrm{TeV} \text {. }
$$

This limit (at 95\% C.L.) is for a genuine left-right symmetric model which we consider here (MLRSM) with $g_{L}=g_{R}$ and three degenerate generations of heavy neutrinos and it is based on $\sqrt{s}=8 \mathrm{TeV}$ data. Typically, also limits for $Z_{2}$ mass are already beyond $2 \mathrm{TeV}$.

The combined LEP lower limit on the singly charged Higgs boson mass is about $90 \mathrm{GeV}[19]$. At the LHC, established limits for singly charged Higgs boson masses are

$$
M_{H^{ \pm}}=80 \div 160 \mathrm{GeV}
$$

if $\mathrm{BR}\left(t \rightarrow H^{+} b\right)<5 \%$ [20] and for higher masses than $160 \mathrm{GeV}$, see the limits in [21].

For doubly charged Higgs bosons the analysis gives lower mass limits in a range

$$
M_{H^{ \pm \pm}} \geq 445 \mathrm{GeV}(409 \mathrm{GeV}) \text { for CMS (ATLAS), }
$$

in the $100 \%$ branching fraction scenarios $[22,23]$.

The mass limit for heavy neutrinos is $[24,25]$

$$
M_{N_{R}}>780 \mathrm{GeV}
$$

but it must be kept in mind that bounds on $M_{N_{R}}$ and $M_{W_{2}}$ are not independent from each other $[17,18]$. Neutrinoless double beta decay allows for heavy neutrinos with relatively light masses, see e.g. [26-32]. Detailed studies which take into account potential signals with $\sqrt{s}=14 \mathrm{TeV}$ at the LHC conclude that heavy gauge bosons and neutrinos can be found with up to 4 and $1 \mathrm{TeV}$, respectively, for typical LR scenarios [3, 4]. Such a relatively low $(\mathrm{TeV})$ scale of the heavy sector is theoretically possible, even if GUT gauge unification is demanded, for a discussion, see e.g. [33, 34].

In this paper we consider Left-Right symmetric model based on the $\mathrm{SU}(2)_{L} \otimes \mathrm{SU}(2)_{R} \otimes$ $\mathrm{U}(1)_{B-L}$ gauge group [1] in its most restricted form, so-called Minimal Left-Right Symmetric Model (MLRSM). We choose to explore the most popular version of the model with Higgs representations - a bi-doublet $\Phi$ and two (left and right) triplets $\Delta_{L, R}[35,36]$. We also assume that the vacuum expectation value of the left-handed triplet $\Delta_{L}$ vanishes, $\left\langle\Delta_{L}\right\rangle=0$ and the CP symmetry can be violated by complex phases in the quark and lepton mixing matrices. Left and right gauge couplings are chosen to be equal, $g_{L}=g_{R}$. For reasons discussed in [37] and more extensively in [38], we discuss see-saw diagonal light-heavy neutrino mixings. It means that $W_{1}$ couples mainly to light neutrinos, while $W_{2}$ couples to the heavy ones. $Z_{1}$ and $Z_{2}$ turn out to couple to both of them [36, 39]. $W_{L}-W_{R}$ mixing is allowed and is very small, $\xi \leq 0.05$ [10], the most stringent data comes from astrophysics through the supernova explosion analysis [40]. In our last paper we considered low energy constraints on such a model assuming $\kappa_{2}=0$, i.e., $\xi=0$ [7], we do the same here. Moreover, in MLRSM $\tan 2 \xi=-\frac{2 \kappa_{1} \kappa_{2}}{v_{R}^{2}}$, which is really negligible for $v_{R} \geq 5 \mathrm{TeV}$, as dictated by eq. (1.1), where $\kappa_{1}, \kappa_{2}\left(v_{R}\right)$ are the vacuum expectation values of $\Phi\left(\Delta_{R}\right)$. 
We think that it is worth to show how the situation looks like if we stick to the popular and to a large extent conservative version of the model (MLRSM), giving candlelike benchmark numbers for possible signals at the LHC. We should also be aware of the fact, that there are relations between model parameters in the Higgs, gauge and neutrino sectors $[7,11,36,41]$ and it needs further detailed studies. For estimation and discussion of observables which are able to measure final signals in the most efficient way, calculation of dominant tree level signals is sufficient at the moment. Production processes are calculated and relevant diagrams are singled out using CalcHEP [42]. For general analysis, multi lepton codes ALPGEN [43], PYTHIA [44], Madgraph [45] are used. Feynman rules are generated with our version of the package using FeynRules [46, 47]. The backgrounds for multi lepton signals (3 and 4 leptons) are estimated using ALPGEN-PYTHIA.

In this paper we have grabbed the impact of the relatively light charged scalars in the phenomenology of Left-Right symmetric model. We first discuss how the decay branching ratios of $W_{2}, Z_{2}$, and $N_{R}$ are affected by the presence of these light charged scalars. Then we note down the possible interesting processes within MLRSM. We study the production and decay modes of the charged scalars. We have provided some benchmark points where we have performed our simulations to make a realistic estimation of the signal events over the SM backgrounds. Our study is based on the reconstruction of the invariant masses of the final state leptons and their mutual separations from where we have shown how we can track the presence of doubly charged scalars. We also note down the impact of the charged scalars in the Higgs to di-photon decay rates. Then we conclude and give an outlook.

\section{MLRSM processes with charged Higgs boson particles at the LHC}

There are already severe limits on the heavy gauge boson masses, eq. (1.1), which infer that scale in which the right $\mathrm{SU}(2)$ gauge sector is broken at $v_{R}>5 \mathrm{TeV}$ (for approximate relations between gauge boson masses and $v_{R}$, see for example eq. (2.4) in [7]). This is already an interesting situation as for such heavy gauge bosons most of the effects connected with them decouple in physical processes at collider physics. Then there is a potential room to go deeper and estimate more sensitive Higgs boson contributions. Of course, the effects coming from the scalar sector depend crucially also on their masses. Smaller the Higgs boson masses, larger effects are expected. The question is then: how small their masses can be by keeping the right scale $v_{R}$ large? In the paper we assume light charged scalar masses up to $600 \mathrm{GeV}$, this choice of masses will be justified when production cross sections are considered.

The point is that all Higgs scalars are naturally of the order of $v_{R}$, in addition, neutral Higgs boson scalars $A_{1}^{0}$ and $H_{1}^{0}$ contribute to FCNC effects (see the appendix) and must be large, above $10 \mathrm{TeV}$ (see however [48] for alternative solutions). Let us see then if theoretically charged Higgs bosons can have masses below $1 \mathrm{TeV}$. In the model which we consider in this paper we assume that the Higgs potential is given as in $[35,36]$, we will also use the same notation, for details on the parametrisation of the Higgs scalar mass spectrum, see the appendix. This model includes a number of parameters: $\mu_{1}, \mu_{2}, \mu_{3}, \rho_{1}, \rho_{2}, \rho_{3}, \rho_{4}$, $\alpha_{1}, \alpha_{2}, \alpha_{3}, \alpha_{4}, \lambda_{1}, \lambda_{2}, \lambda_{3}, \lambda_{4}$. The exact Higgs mass spectrum is calculated numerically. 


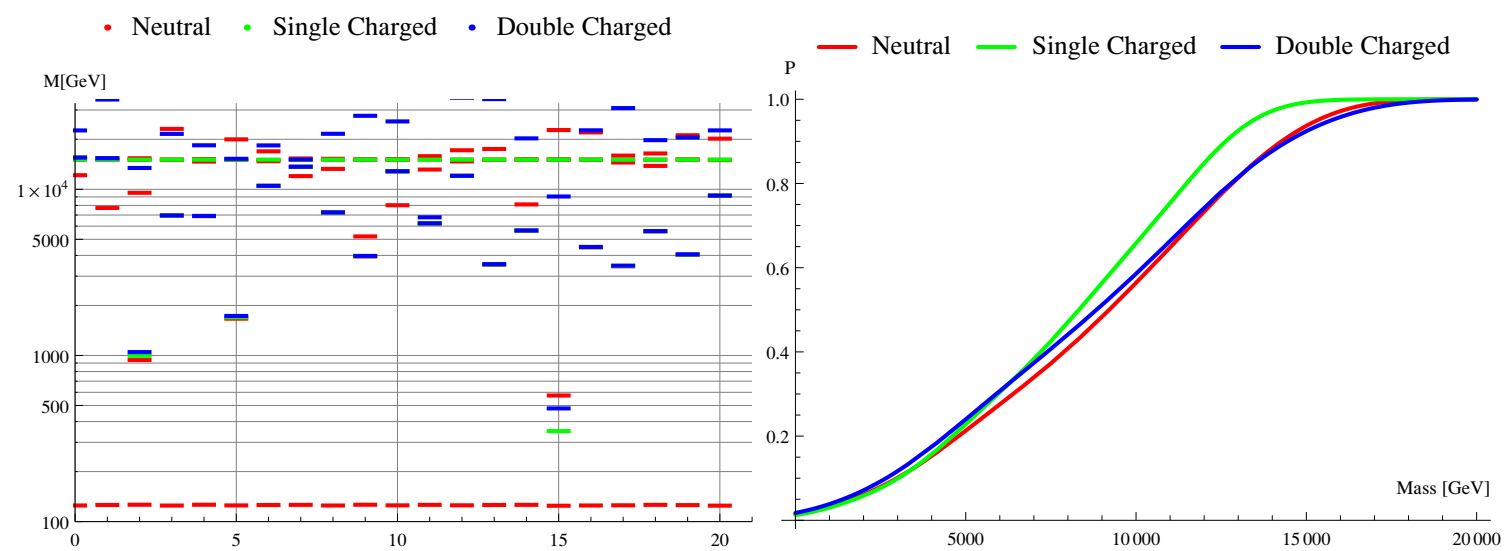

Figure 1. On left: an example of 20 Higgs mass spectra obtained by randomly chosen Higgs potential parameters. The constrain on the lowest neutral Higgs mass eq. (2.1) was imposed and the bounds coming from FCNC were taken into account. On right: cumulative distribution function $P$ of the lowest mass of singly and doubly charged and next to lightest neutral scalars. For both figures, $v_{R}=8 \mathrm{TeV}$.

Minimisation conditions are used to get values of dimensionful mass parameters $\mu_{1}, \mu_{2}$ and $\mu_{3}$ which can be arbitrarily large, all other parameters are considered as free, but limited to the perturbative bound, ${ }^{1}\left|\rho_{i}\right|,\left|\alpha_{i}\right|,\left|\lambda_{i}\right|<10$. It is assumed that the lightest neutral Higgs particle is the boson discovered by ATLAS and CMS collaborations. We have taken its mass to lie in the range

$$
\text { 124.7 GeV }<M_{H_{0}^{0}}<126.2 \mathrm{GeV} .
$$

An example set of generated mass spectra of Higgs bosons for $v_{R}=8 \mathrm{TeV}$ is presented in figure 1 (left figure). Mass spectra have been obtained by varying uniformly the Higgs potential parameters in a range $(-10,10)$. We have also taken into account the bounds on neutral Higgs bosons obtained from FCNC constrains assuming $m_{A_{1}^{0}}, m_{H_{1}^{0}}>15 \mathrm{TeV}$ by fixing $\alpha_{3}=7.1$ (see appendix A). The spectra which did not fulfill relation (2.1) were rejected. Altogether we have 6 neutral, 2 singly charged and 2 doubly charged Higgs boson particles in the MLRSM. The figure includes possible spectra of singly and doubly charged as well as neutral Higgs bosons. Some of them can be degenerated or nearly degenerated.

This study shows that although the Higgs particles naturally tend to have masses of the order of the $v_{R}$ scale, it is still possible to choose the potential parameters such that some of the scalar particles can have masses much below $1 \mathrm{TeV}$ (spectrum 15). To discuss spectra more quantitatively, the cumulative distribution function $P$ of the lowest masses of singly and doubly charged and next to lightest neutral scalar particles are plotted on right figure 1 , again for the same conditions as before and $v_{R}=8 \mathrm{TeV}$. These results show that for $v_{R}=8 \mathrm{TeV}$ a fraction of the parameter space that gives lightest scalar masses below $1 \mathrm{TeV}$ is at the level of $4 \%$. It means that it is possible to generate the low mass spectra of Higgs boson masses in MLRSM keeping large $v_{R}$ scale. However, what can not be seen

\footnotetext{
${ }^{1}$ Which is equal to $4 \pi$, otherwise proper analysis of the Higgs potential with radiative corrections to determine perturbative regions would be needed.
} 


\begin{tabular}{|c|c|c|}
\hline Primary production & Secondary production & Signal \\
\hline I. $H_{1}^{+} H_{1}^{-}$ & $\ell^{+} \ell^{-} \nu_{L} \nu_{L}$ & $\ell^{+} \ell^{-} \oplus M E T$ \\
\hline- & $\ell^{+} \ell^{-} N_{R} N_{R}$ & depends on $N_{R}$ decay modes \\
\hline- & $\ell^{+} \ell^{-} \nu_{L} N_{R}$ & depends on $N_{R}$ decay modes \\
\hline II. $H_{2}^{+} H_{2}^{-}$ & $\ell^{+} \ell^{-} \nu_{L} \nu_{L}$ & $\ell^{+} \ell^{-} \oplus M E T$ \\
\hline- & $\ell^{+} \ell^{-} N_{R} N_{R}$ & depends on $N_{R}$ decay modes \\
\hline- & $\ell^{+} \ell^{-} \nu_{L} N_{R}$ & depends on $N_{R}$ decay modes \\
\hline III. $H_{1}^{++} H_{1}^{--}$ & - & $\ell^{+} \ell^{+} \ell^{-} \ell^{-}$ \\
\hline- & $H_{1}^{+} H_{1}^{+} H_{1}^{-} H_{1}^{-}$ & See I \\
\hline- & $H_{1}^{ \pm} H_{1}^{ \pm} H_{2}^{\mp} H_{2}^{\mp}$ & See I \& II \\
\hline- & $H_{2}^{+} H_{2}^{+} H_{2}^{-} H_{2}^{-}$ & See II \\
\hline- & $W_{i}^{+} W_{i}^{+} W_{j}^{-} W_{j}^{-}$ & depends on $W^{\prime}$ s decay modes \\
\hline IV. $H_{2}^{++} H_{2}^{--}$ & - & $\ell^{+} \ell^{+} \ell^{-} \ell^{-}$ \\
\hline- & $H_{2}^{+} H_{2}^{+} H_{2}^{-} H_{2}^{-}$ & See II \\
\hline- & $H_{1}^{ \pm} H_{1}^{ \pm} H_{2}^{\mp} H_{2}^{\mp}$ & See I \& II \\
\hline- & $H_{1}^{+} H_{1}^{+} H_{1}^{-} H_{1}^{-}$ & See I \\
\hline- & $W_{i}^{+} W_{i}^{+} W_{j}^{-} W_{j}^{-}$ & depends on $W^{\prime}$ 's decay modes \\
\hline V. $H_{1}^{ \pm \pm} H_{1}^{\mp}$ & - & $\ell^{ \pm} \ell^{ \pm} \ell^{\mp} \nu_{L}$ \\
\hline VI. $H_{2}^{ \pm \pm} H_{2}^{\mp}$ & - & $\ell^{ \pm} \ell^{ \pm} \ell^{\mp} \nu_{L}$ \\
\hline VII. $H_{1}^{ \pm} Z_{i}, H_{1}^{ \pm} W_{i}$ & - & See I \& $Z_{i}, W_{i}$ decay modes \\
\hline VIII. $H_{2}^{ \pm} Z_{i}, H_{2}^{ \pm} W_{i}$ & - & See II $\& Z_{i}, W_{i}$ decay modes \\
\hline IX. $H_{1}^{ \pm} \gamma$ & - & See I \\
\hline $\mathrm{X} . H_{2}^{ \pm} \gamma$ & - & See II \\
\hline
\end{tabular}

Table 1. Phenomenologically interesting MLRSM processes at the LHC with primarily produced charged scalar particles and possible final signals. Here $\gamma$ denotes a photon. $\nu_{L}=\nu_{1}, \nu_{2}, \nu_{3}$ are SM-like light massive neutrino states and $N_{R}=N_{4,5,6}$ are heavy neutrino massive states dominated by right-handed weak neutrinos. From now on we will denote $N_{R} \equiv N$. Here $\ell$ represents light charged leptons $e, \mu$.

on those plots is that in MLRSM not all four charged Higgs bosons can simultaneously be light. It is a case for $H_{1}^{ \pm}, H_{1}^{ \pm \pm}$and $H_{2}^{ \pm \pm}$, for details, see the appendix. The remaining charged scalar $H_{2}^{ \pm}$is of the order of the $v_{R}$ scale, so its effects at LHC is negligible, to make it lighter would require to go beyond MLRSM. For a book keeping, we keep this particle in further discussion. If its mass at some points is assumed to be small (so we go beyond MLRSM), we denote it with a tilde, $\tilde{H}_{2}^{ \pm}$. Its coupling is kept all the time as in MLRSM (why it can be so is discussed shortly in the appendix).

In this paper we consider only the processes where charged Higgs particles can be produced directly as shown in the table 1, first column.

The decay branching ratios for heavy neutrino states $N$ and heavy gauge bosons $\left(W_{2}, Z_{2}\right)$ in MLRSM which determine both secondary production and final signals in the last column of this table are given in [7]. However, with assumed light charged 

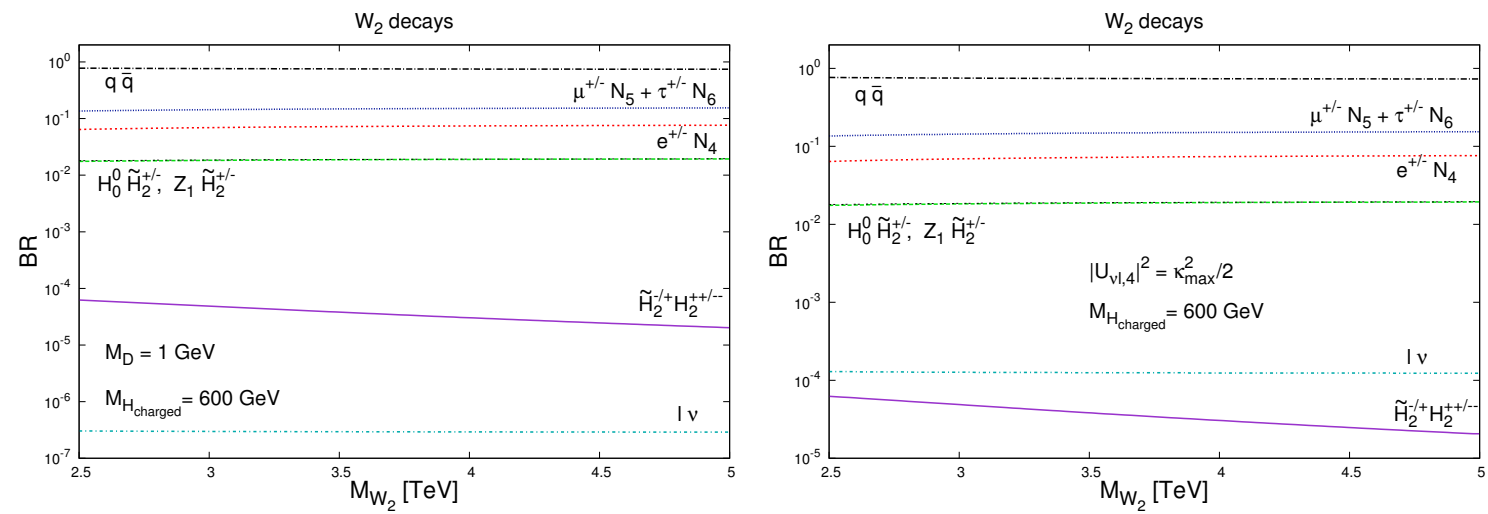

Figure 2. Branching ratio for $W_{2}$ decay with relatively light charged scalars. Here we put $M_{N_{4}}=$ $M_{N_{5}}=1 \mathrm{TeV}, M_{N_{6}}=800 \mathrm{GeV}$. Symbol $q \bar{q}$ on this and next plots stands for a sum of all quark flavours, $q \bar{q} \equiv \sum_{i, i^{\prime}=u, d, s, b, c, t} q_{i} \overline{q_{i^{\prime}}}$. Similarly, $l \nu \equiv \sum_{i=1}^{3} l_{i} \nu_{i}$.
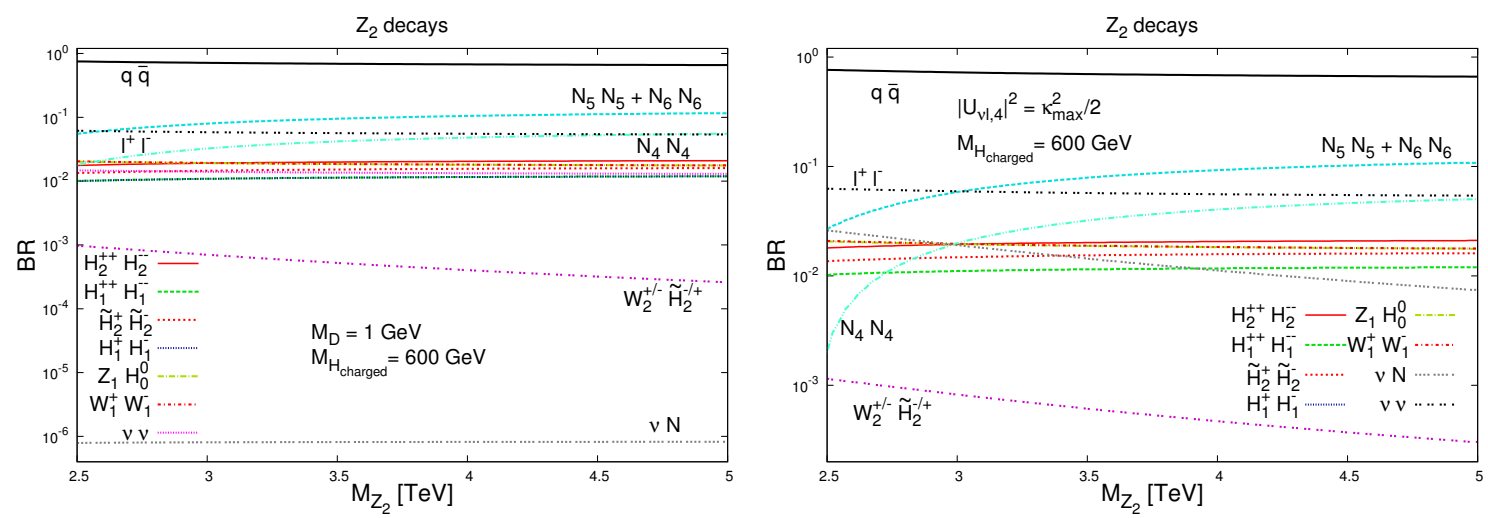

Figure 3. Branching ratio for $Z_{2}$ decay with relatively light charged scalars. Here $\nu \nu \equiv \sum_{i=1}^{3} \nu_{i} \nu_{i}$ and $\nu N \equiv \sum_{i=1}^{3} \nu_{i} N_{i+3}$.

Higgs particles, new decay modes are potentially open, and discussion must be repeated. Results are given in figures 2, 3, and 4. As can be seen from figure 2, contribution of charged scalars to the total decay width of $W_{2}$ is at the percent level. Here more important are heavy neutrino decay modes. ${ }^{2}$ Different scenarios for LH neutrino mixings [7] are discussed, i.e., see-saw mechanisms where $\left|U_{\nu_{i} j}\right| \simeq \frac{\left|\left\langle M_{D}\right\rangle\right|}{M_{N_{j}}} \delta_{i, j-3}, i=1,2,3$, $j=4,5,6$ and scenarios where LH neutrino mixings are independent of neutrino masses: $\sum_{j=4,5,6} U_{\nu_{1}, j-3} U_{\nu_{1}, j-3}^{*}=U_{\nu_{1}, 4} U_{\nu_{1}, 4}^{*} \leq 0.003 \equiv \kappa_{\max }^{2}$ [49]. In a case of many heavy neutrino states (as in MLRSM), taking into account constraints coming from neutrinoless double-beta decay experiment, this limit becomes $\kappa_{\max }^{2} / 2$ [50-52]. For $W_{2}$ decays different LH neutrino mixing scenarios affect only light neutrino $\nu l$ channel for which BR is small, anyway.

For the $Z_{2}$ decays, figure 3, four channels with charged Higgs bosons, namely $H_{1}^{++} H_{1}^{--}$, $H_{1}^{+} H_{1}^{-}, H_{2}^{++} H_{2}^{--}$, and $\tilde{H}_{2}^{+} \tilde{H}_{2}^{-}$, contribute to the decay rate in a percentage level. The

\footnotetext{
${ }^{2}$ Some processes in the table 1 depend strongly on the light-heavy (LH) neutrino mixing scenarios.
} 

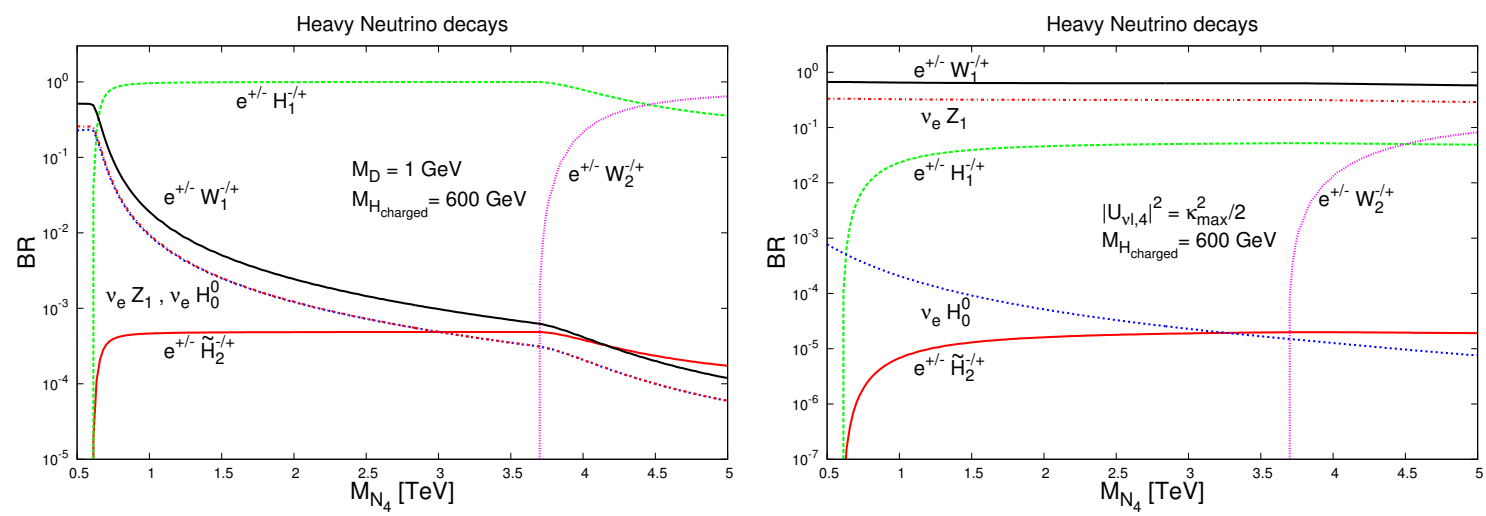

Figure 4. Branching ratios for $N_{4}$ decay with relatively light charged scalars.

quark decay modes dominate, and the second important are the heavy neutrino decay modes.

The most interesting situation is for the decays of heavy neutrinos. Here $H_{1}^{+}$decay mode is the largest in see-saw scenarios. The reason is that in case of Yukawa coupling, say $H_{1}^{+}-N-e$, the change in LH neutrino mixing is compensated by the proportionality of the coupling to the heavy neutrino mass, which is not the case for the gauge $N-e-W$ and $N-\nu-Z$ couplings. That is why $e W$ and $\nu Z$ decay modes are relevant only in scenarios where LH neutrino mixings are independent of the heavy neutrino masses and are close to the present experimental limits. Large charged Higgs boson decay mode of the heavy neutrino can influence the "golden" $p p \rightarrow e N$ process [3, 4, 6, 7, 53, 54].

For typical see-saw cases when charged Higgs boson masses are very large, standard model modes dominate: $N \rightarrow e W_{1}$ and $N \rightarrow \nu_{L} Z_{1}$ if $M_{N}<M_{W_{2}}$ whereas $N \rightarrow e W_{2}$ if $M_{N}>M_{W_{2}}$. In scenarios with large LH neutrino mixings the standard modes dominates independently of the heavy neutrino and $W_{2}$ masses. ${ }^{3}$ Finally, let us note that in typical Type I see-saw scenarios the TeV scale of heavy neutrino masses implies $m_{D} \sim 10^{-6} \mathrm{GeV}$ to accomplish light neutrino masses at the $\mathrm{eV}$ level. In this situation nothing happens to the left plots in figures 2, 3, and 4 apart from the fact that $l \nu, l N$ and $\nu Z$ channels will disappear completely there.

In the case of heavy gauge boson decays, quarks dominate and jets will be produced while for SM-like gauge bosons hadronic decay branching is around $70 \%$. That is why typical final signals for reactions I and II in table 1 are two or four jets plus missing energy. There are only two cases without missing energy:

$$
H_{1(2)}^{+} H_{1(2)}^{-} \rightarrow \ell^{+} \ell^{-} N N \rightarrow \ell^{+} \ell^{-} W_{m}^{ \pm} \ell^{\mp} W_{n}^{ \pm} \ell^{\mp} \rightarrow j j j j \ell^{+} \ell^{-} \ell^{\mp} \ell^{\mp},
$$

and

$$
H_{1(2)}^{+} H_{1(2)}^{-} \rightarrow \ell^{+} \ell^{-} N N \rightarrow \ell^{+} \ell^{-} W_{m}^{ \pm} \ell^{\mp} W_{n}^{\mp} \ell^{ \pm} \rightarrow j j j j \ell^{+} \ell^{-} \ell^{ \pm} \ell^{\mp} .
$$

However, as we can see from the table, the cleanest signals are connected with doubly charged Higgs particles, that is why we focus on them in this paper. For some related

\footnotetext{
${ }^{3}$ Relevance of see-saw LH mixings at the LHC has been discussed lately in [54].
} 


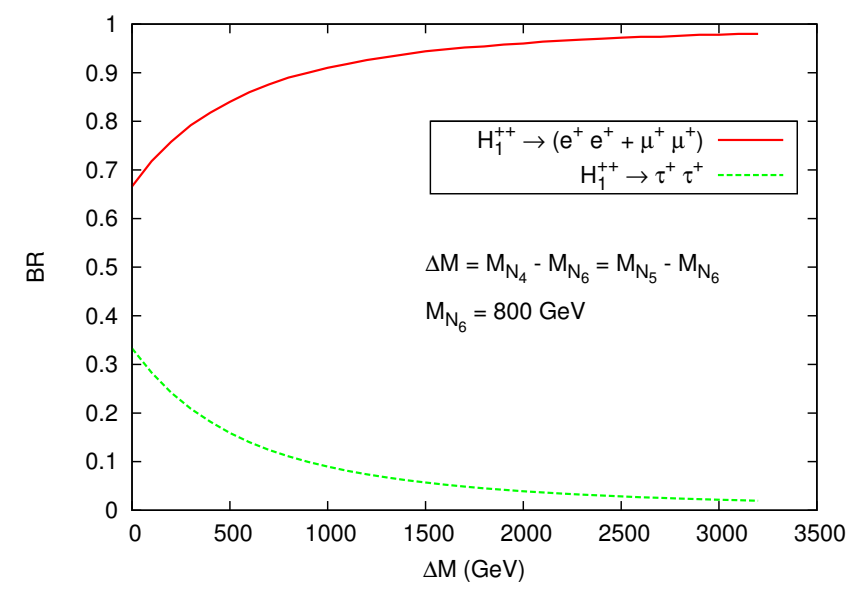

Figure 5. Branching ratios for the decay modes $\left(e^{+} e^{+}+\mu^{+} \mu^{+}\right)$and $\tau^{+} \tau^{+}$of the doubly charged scalars as a function of $\Delta M$, where $\Delta M=M_{N_{4}}-M_{N_{6}}=M_{N_{5}}-M_{N_{6}}$. We have kept fixed $M_{N_{6}}=800 \mathrm{GeV}$. Note that the BRs of both the doubly charged scalars $\left(H_{1}^{++}\right.$and $\left.H_{2}^{++}\right)$are the same in scenarios where $M_{W_{2}} \gg M_{H^{ \pm \pm}}$and $M_{H^{ \pm \pm}} \simeq M_{H^{ \pm}}$.

discussions on doubly charged scalars, see e.g. [55-63]. The processes eqs. (2.2) and (2.3) with four charged leptons plus jets will be considered elsewhere.

For processes III-X important are charged Higgs boson decay modes. For doubly charged Higgs particles possible decay modes are

$$
\begin{aligned}
& \text { (i) } \quad H_{1}^{ \pm \pm} \rightarrow l^{ \pm} l^{ \pm}, \\
& \text {(ii) } \quad H_{1}^{ \pm \pm} \rightarrow H_{1}^{ \pm} W_{1}^{ \pm} ; \\
& \text {(iii) } \quad H_{2}^{ \pm \pm} \rightarrow l^{ \pm} l^{ \pm}, \\
& \text {(iv) } H_{2}^{ \pm \pm} \rightarrow H_{2}^{ \pm} W_{2}^{ \pm} ; \\
& \text {(v) } H_{2}^{ \pm \pm} \rightarrow W_{2}^{ \pm} W_{2}^{ \pm} ; \\
& \text {(vi) } H_{2}^{ \pm \pm} \rightarrow H_{2}^{ \pm} W_{1}^{ \pm} ;
\end{aligned}
$$

where $l=e, \mu, \tau$.

Apart from the above decay modes, the other possibilities for the doubly charged scalars can be

$$
\begin{aligned}
& \text { (vii) } \quad H_{2}^{ \pm \pm} \rightarrow H_{1}^{ \pm} H_{1}^{ \pm}, \\
& \text {(viii) } \quad H_{2}^{ \pm \pm} \rightarrow H_{2}^{ \pm} H_{2}^{ \pm} ;
\end{aligned}
$$

when they are not degenerate with the singly charged ones. But for nearly or exact degenerate case, the charged scalars dominantly decay through leptonic modes and here kinematics play a role too.

Figure 5 shows a scenario in which pure leptonic decay modes can be realised. The crucial factor is the Yukawa coupling which depends (indirectly) on heavy right-handed neutrino mass. If heavy neutrino masses are degenerate then democratic scenario is understood where all leptonic channels are the same (i.e. $\operatorname{BR}\left(H^{ \pm \pm} \rightarrow e^{ \pm} e^{ \pm}\right) \simeq 33 \%$ ). 
Typically, as can be seen from figure 5 , for right-handed neutrino masses to be $1 \mathrm{TeV}$, $1 \mathrm{TeV}$ and $800 \mathrm{GeV}$ for $N_{4}, N_{5}, N_{6}$ respectively, the branching ratios are the following

$$
\begin{aligned}
\operatorname{BR}\left(H_{1 / 2}^{ \pm \pm} \rightarrow e^{ \pm} e^{ \pm}\right) & =37.9 \% \\
\operatorname{BR}\left(H_{1 / 2}^{ \pm \pm} \rightarrow \mu^{ \pm} \mu^{ \pm}\right. & =37.9 \% \\
\operatorname{BR}\left(H_{1 / 2}^{ \pm \pm} \rightarrow \tau^{ \pm} \tau^{ \pm}\right. & =24.2 \%
\end{aligned}
$$

If the first two generations neutrinos $\left(N_{4}, N_{5}\right)$ have masses above $\sim 4 \mathrm{TeV}, \tau$ decay mode is practically irrelevant. From the discussion it is also clear, that one of the decay modes can dominate if only one of the right-handed neutrino masses is much bigger than remaining two heavy neutrino states. Limits in eq. (1.3) assume $100 \%$ leptonic decays, in our case, taking into account figure 5, eq. (2.6) and results given in [22, 23], mass limits are much weaker, at about $300 \mathrm{GeV}$, see e.g. figure 3 in [23].

For decays of singly charged $H_{1}^{ \pm}$scalars situation is analogical as for doubly charged scalars (possible decay modes to neutral $H_{1}^{0}$ and $A_{1}^{0}$ scalars are negligible for $M_{H_{1}^{0}}, M_{A_{1}^{0}} \gg$ $M_{H_{1}^{ \pm}}$, as dictated by FCNC constraints).

$\tilde{H}_{2}^{ \pm}$decays hadronicaly, namely, for $100 \mathrm{GeV}<M_{\tilde{H}_{2}^{ \pm}}<200 \mathrm{GeV}$

$$
\begin{aligned}
& \operatorname{BR}\left(\tilde{H}_{2}^{+} \rightarrow c \bar{s}\right)=95 \%, \\
& \operatorname{BR}\left(\tilde{H}_{2}^{+} \rightarrow c \bar{d}\right)=5 \%,
\end{aligned}
$$

and $\operatorname{BR}\left(\tilde{H}_{2}^{+} \rightarrow t \bar{b}\right) \sim 100 \%$ for $M_{\tilde{H}_{2}^{ \pm}}>200 \mathrm{GeV}$.

\subsection{Primary production of heavy charged Higgs bosons at the LHC}

Below different processes involving solely charged scalar productions are classified. In analysis which follow $v_{R}=8000 \mathrm{GeV}$ to respect with a large excess the present exclusion limits on $W_{2}^{ \pm}$, and $Z_{2}$ masses. SM-Higgs like mass is set to $125 \mathrm{GeV}$, masses of neutral scalar particles are set at very high limit $(\sim 10 \mathrm{TeV})$. In this way, as already discussed, scenarios are realised with relatively light (hundreds of $\mathrm{GeV}$ ) charged Higgs bosons while remaining non-standard particles within MLRSM are much heavier. All cross sections given in this section are without any kinematic cuts, those will be considered with final signals and distributions in section 3.

\subsection{1 $p p \rightarrow H_{1}^{+} H_{1}^{-}$and $p p \rightarrow H_{2}^{+} H_{2}^{-}$}

The cross section for singly charged scalar pair production as a function of their mass is given in figure 6. This process is dominated by s-channel $\gamma, Z_{1}$ and t-channel quark exchange diagrams. Contributions coming from s-channel $H_{0}^{0}, Z_{2}$ and $H_{1}^{0}$ bosons are negligible for considered MLRSM parameters. For singly charged scalar mass equals to $400 \mathrm{GeV}$, the cross sections are (as discussed in section 2, $\mathrm{H}_{2}^{ \pm}$Higgs boson is assumed to be light and we denote it here with a tilde, for $M_{H_{2}^{ \pm}} \gg 1 \mathrm{TeV}$ the considered cross section is negligible, $\left.\sigma\left(p p \rightarrow \tilde{H}_{2}^{ \pm} \tilde{H}_{2}^{\mp}\right) \simeq 0\right)$

$$
\begin{aligned}
\sigma\left(p p \rightarrow H_{1}^{ \pm} H_{1}^{\mp}\right) & =0.12(0.52) \mathrm{fb}, \\
\sigma\left(p p \rightarrow \tilde{H}_{2}^{ \pm} \tilde{H}_{2}^{\mp}\right) & =0.27(1.12) \mathrm{fb},
\end{aligned}
$$




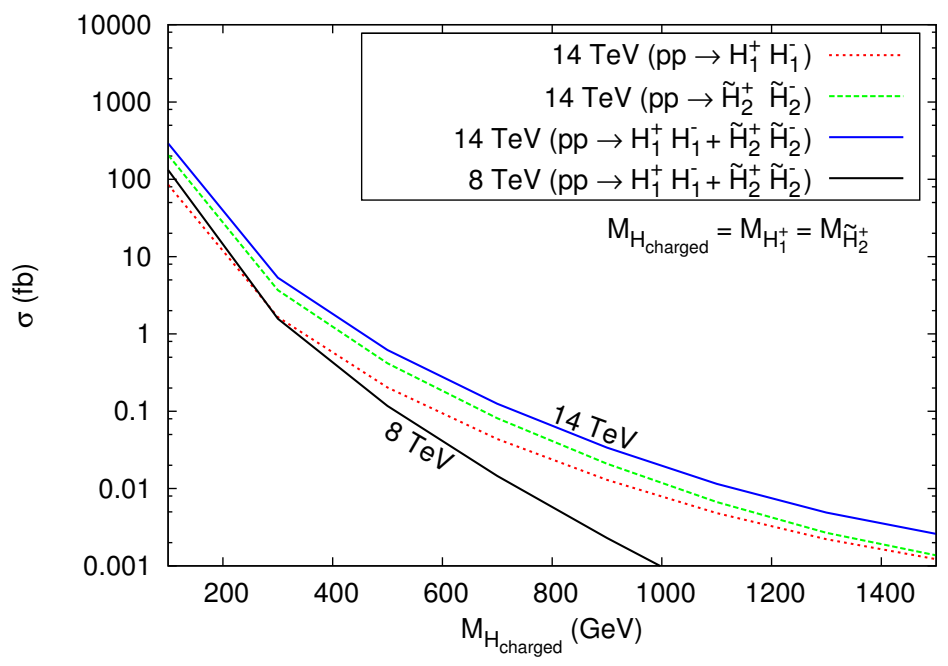

Figure 6. Production cross sections for $p p \rightarrow H_{1}^{+} H_{1}^{-}$and $p p \rightarrow \tilde{H}_{2}^{+} \tilde{H}_{2}^{-}$processes without imposing kinematic cuts.

while for singly charged scalar mass equals to $600 \mathrm{GeV}$ are

$$
\begin{aligned}
\sigma\left(p p \rightarrow H_{1}^{ \pm} H_{1}^{\mp}\right) & =0.01(0.09) \mathrm{fb}, \\
\sigma\left(p p \rightarrow \tilde{H}_{2}^{ \pm} \tilde{H}_{2}^{\mp}\right) & =0.03(0.18) \mathrm{fb},
\end{aligned}
$$

with $\sqrt{s}=8(14) \mathrm{TeV}$.

Increasing center of mass energy from $\sqrt{s}=8 \mathrm{TeV}$ to $\sqrt{s}=14 \mathrm{TeV}$ the cross sections grow by factors $\sim 4 \div 7$, depending on masses of charged Higgs bosons. In general cross sections fall down below $0.1 \mathrm{fb}$ for masses of charged scalars above approximately $730(420) \mathrm{GeV}$ for $\sqrt{s}=14(8) \mathrm{TeV}$.

\subsection{2 $\quad p p \rightarrow H_{1}^{++} H_{1}^{--}$and $p p \rightarrow H_{2}^{++} H_{2}^{--}$}

The dominant contribution to these processes is via neutral s-channel current, i.e., via $Z_{1}$ and $\gamma$. Contributions coming from s-channel $H_{0}^{0}, Z_{2}$ and $H_{1}^{0}$ are negligible for considered MLRSM parameters.

To explore the phenomenological aspects of the doubly charged scalars in the MLRSM model we consider two scenarios. Scenario $I$ when the doubly charged scalars are degenerated in mass, i.e., $M_{H_{1}^{ \pm \pm}}=M_{H_{2}^{ \pm \pm}}$. This scenario is motivated by analysis of the Higgs potential (a detailed study of the Higgs potential and scalar mass spectrum will be presented elsewhere). In Scenario II masses are different, i.e., $M_{H_{1}^{ \pm \pm}} \neq M_{H_{2}^{ \pm \pm}}$.

Scenario I, degenerate mass spectrum. In our analysis we set our benchmark point with both of the doubly charged scalars at the same mass $M_{H_{1}^{++}}=M_{H_{2}^{++}}=400 \mathrm{GeV}$. In this case, the cross section at the LHC without imposing any cut at $\sqrt{s}=8(14) \mathrm{TeV}$ is

$$
\sigma\left(p p \rightarrow\left(H_{1}^{++} H_{1}^{--}+H_{2}^{++} H_{2}^{--}\right) \rightarrow \ell_{i}{ }^{+} \ell_{i}{ }^{+} \ell_{j}{ }^{-} \ell_{j}{ }^{-}\right)=1.44(6.06) \mathrm{fb} .
$$




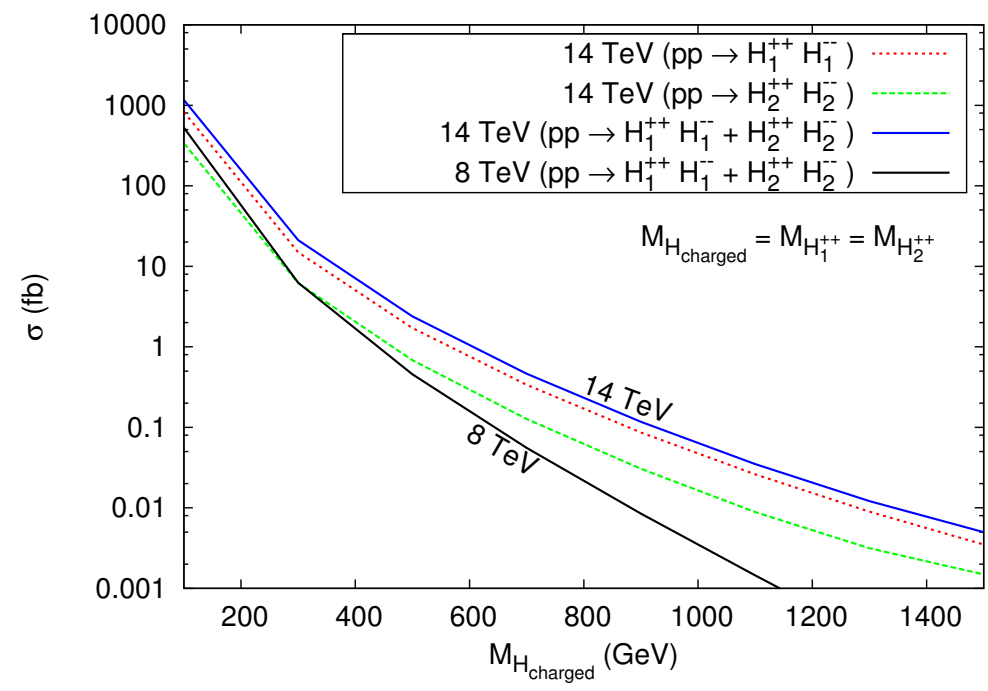

Figure 7. Scenario I. Cross sections for $p p \rightarrow H_{1}^{++} H_{1}^{--}$and $p p \rightarrow H_{2}^{++} H_{2}^{--}$processes without imposing kinematic cuts.

The contributions to the cross sections from two possible channels are noted for $\sqrt{s}=$ $8(14) \mathrm{TeV}$ as

$$
\begin{aligned}
& \sigma\left(p p \rightarrow H_{1}^{++} H_{1}^{--}\right)=1.09(4.58) \mathrm{fb}, \\
& \sigma\left(p p \rightarrow H_{2}^{++} H_{2}^{--}\right)=0.45(1.86) \mathrm{fb},
\end{aligned}
$$

where $\ell_{i, j}=e, \mu$.

For $M_{H_{1}^{++}}=M_{H_{2}^{++}}=600 \mathrm{GeV}$ it is

$$
\sigma\left(p p \rightarrow\left(H_{1}^{++} H_{1}^{--}+H_{2}^{++} H_{2}^{--}\right) \rightarrow \ell_{i}{ }^{+} \ell_{i}{ }^{+} \ell_{j}{ }^{-} \ell_{j}^{-}\right)=0.14(0.95) \mathrm{fb},
$$

for $\sqrt{s}=8(14) \mathrm{TeV}$. The contributions to the cross sections from individual channels for $\sqrt{s}=8(14) \mathrm{TeV}$ are as following:

$$
\begin{aligned}
\sigma\left(p p \rightarrow H_{1}^{++} H_{1}^{--}\right) & =0.11(0.73) \mathrm{fb}, \\
\sigma\left(p p \rightarrow H_{2}^{++} H_{2}^{--}\right) & =0.04(0.28) \mathrm{fb} .
\end{aligned}
$$

The cross sections for pair productions of doubly charged scalars at the LHC with 14 and $8 \mathrm{TeV}$ are given in figure 7 . From the figure we can see that cross sections fall very rapidly as the masses of the doubly charged scalars increase. Also the production cross section for $H_{1}^{ \pm \pm}$is much larger than that for $H_{2}^{ \pm \pm}$as shown in the figure. The cross section at $\sqrt{s}=14(8) \mathrm{TeV}$ for scalar masses above $920(640) \mathrm{GeV}$ is $\leq 0.1 \mathrm{fb}$.

Scenario II, non-degenerated mass spectrum. Here we choose another set of benchmark points where the doubly charged scalars are non-degenerated. The cross section for the same process with $M_{H_{1}^{ \pm \pm}}=400 \mathrm{GeV}$ and $M_{H_{2}^{ \pm \pm}}=500 \mathrm{GeV}$ at $\sqrt{s}=14 \mathrm{TeV}$ is

$$
\sigma\left(p p \rightarrow\left(H_{1}^{++} H_{1}^{--}+H_{2}^{++} H_{2}^{--}\right) \rightarrow \ell_{i}{ }^{+} \ell_{i}{ }^{+} \ell_{j}^{-} \ell_{j}^{-}\right)=4.95 \mathrm{fb} .
$$



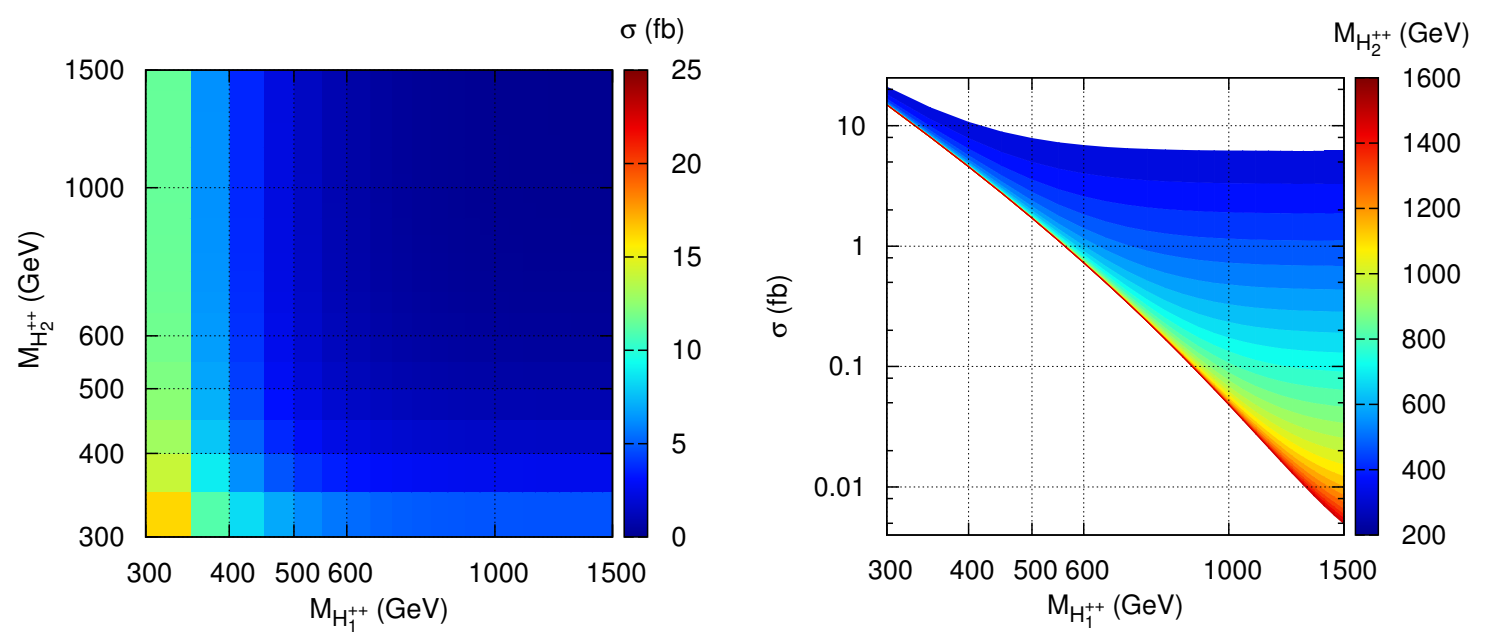

Figure 8. Scenario II. Contour plots for the $p p \rightarrow\left(H_{1}^{++} H_{1}^{--}+H_{2}^{++} H_{2}^{--}\right)$cross section. $\sqrt{s}=$ $14 \mathrm{TeV}$, no kinematic cuts imposed.

The contributions to the cross sections from individual channels are given as:

$$
\begin{aligned}
\sigma\left(p p \rightarrow H_{1}^{++} H_{1}^{--}\right) & =1.09(4.58) \mathrm{fb}, \\
\sigma\left(p p \rightarrow H_{2}^{++} H_{2}^{--}\right) & =0.13(0.69) \mathrm{fb},
\end{aligned}
$$

for $\sqrt{s}=8(14) \mathrm{TeV}$.

Contour plots for the $p p \rightarrow\left(H_{1}^{++} H_{1}^{--}+H_{2}^{++} H_{2}^{--}\right)$cross section as a function of doubly charged scalar masses is shown in figure 8 (left). On the right figure of figure 8 different projections are used where $\mathrm{X}$ and $\mathrm{Y}$ axes are for $M_{H_{1}^{++}}$and the cross section, respectively, whereas $M_{H_{2}^{++}}$is projected as a contour. As can be seen from these figures, cross sections at the level of $1 \mathrm{fb}$ can be obtained for doubly charged scalar masses up to approximately $600 \mathrm{GeV}$.

\subsection{3 $p p \rightarrow H_{1}^{ \pm \pm} H_{1}^{\mp}$ and $p p \rightarrow H_{2}^{ \pm \pm} H_{2}^{\mp}$}

The production of a doubly charged in association with a singly charged scalar goes through the charged s-channel interaction where $W_{1,2}^{ \pm}$gauge bosons are exchanged. Diagrams with schannel exchanged singly charged scalar $H_{2}^{ \pm}$is negligible (its coupling to $W_{1}$ is proportional to $v_{L}$ which is zero). As $W_{2}^{ \pm}$is very heavy, the dominant contribution originates from the process via $W_{1}^{ \pm}$.

To give yet another benchmark, we set $v_{R}=8 \mathrm{TeV}$ and the following charged scalar masses: $M_{H_{1}^{ \pm \pm}}=483 \mathrm{GeV}, M_{H_{2}^{ \pm \pm}}=527 \mathrm{GeV}, M_{H_{1}^{ \pm}}=355 \mathrm{GeV}, M_{H_{2}^{ \pm}}=15066 \mathrm{GeV}$. The choice is for the following Higgs potential parameters (for the mass formulas, see the appendix): $\rho_{1}=0.2397, \rho_{2}=0.0005, \rho_{3}=0.48, \lambda_{1}=0.13, \lambda_{2}=-0.87, \lambda_{3}=-5.17$, $\alpha_{3}=7.09$. This example shows that a wide spectrum of charged scalar masses can be easily obtained, still keeping reasonable small potential parameters (important for higher order perturbation analysis). To reduce $\tau$ channel decays, the masses for the heavy right handed neutrinos are set at $4 \mathrm{TeV}$ for the first two generations and $800 \mathrm{GeV}$ for the third 


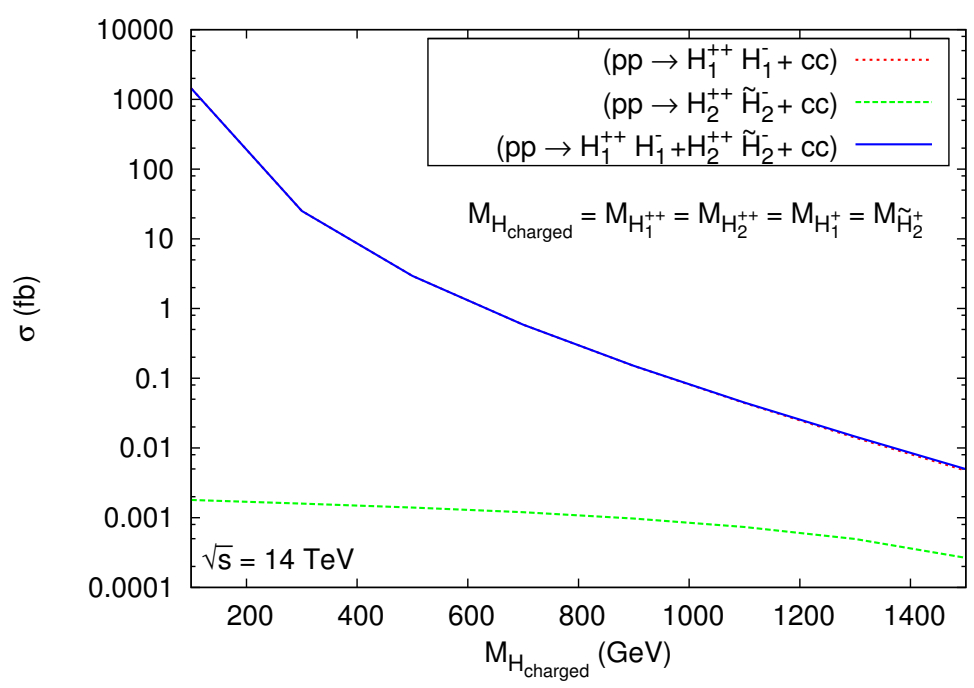

Figure 9. Production cross sections for $p p \rightarrow H_{1}^{++} H_{1}^{-}$and $p p \rightarrow H_{2}^{++} \tilde{H}_{2}^{-}$processes at $\sqrt{s}=$ $14 \mathrm{TeV}$ and no kinematic cuts are imposed. Mass of $H_{2}^{ \pm}$is allowed to be small and denoted with a tilde.

generation, see figure 5. The cross section for the process before any kinematic cuts with centre of mass energy $\sqrt{s}=8(14) \mathrm{TeV}$ at the LHC is

$$
\sigma\left(p p \rightarrow\left(H_{1}^{ \pm \pm} H_{1}^{\mp}+H_{2}^{ \pm \pm} H_{2}^{\mp}\right) \rightarrow \ell \ell \ell \nu_{\ell}\right)=1.44(6.05) \mathrm{fb} .
$$

The contributions to the cross sections from individual channels are noted as:

$$
\begin{aligned}
\sigma\left(p p \rightarrow H_{1}^{ \pm \pm} H_{1}^{\mp}\right) & =1.48(6.24) \mathrm{fb}, \\
\sigma\left(p p \rightarrow H_{2}^{ \pm \pm} H_{2}^{\mp}\right) & \sim 0(0) \mathrm{fb},
\end{aligned}
$$

with $\sqrt{s}=8(14) \mathrm{TeV}$.

For the model consistency (i.e. chosen potential parameters), the second singly charged scalar has been chosen with very high mass $M_{H_{2}^{ \pm}}=15066 \mathrm{GeV}$. Even if it has low mass $(\sim$ $400 \mathrm{GeV}$ ) then also the cross section for the processes $p p \rightarrow H_{2}^{ \pm \pm} H_{2}^{\mp}$ is very low compared to $p p \rightarrow H_{1}^{ \pm \pm} H_{1}^{\mp}$ as $H_{2}^{ \pm \pm} H_{2}^{\mp} W_{1}^{\mp}$ coupling is proportional to $\sin \xi$ and $H_{2}^{ \pm \pm} H_{2}^{\mp} W_{2}^{\mp}$ coupling is proportional to $\cos \xi$. On the other hand, $H_{1}^{ \pm \pm} H_{1}^{\mp} W_{1}^{\mp}$ coupling is proportional to $\cos \xi$ and $H_{1}^{ \pm \pm} H_{1}^{\mp} W_{2}^{\mp}$ coupling is proportional to $\sin \xi$. In both cases $W_{2}^{ \pm}$mediated processes are much less dominant than the $W_{1}^{ \pm}$mediated processes. But as the charged gauge boson mixing angle $\xi$ is neglected, the $H_{2}^{ \pm \pm} H_{2}^{\mp} W_{1}^{\mp}$ vertex is much more suppressed compare to $H_{1}^{ \pm \pm} H_{1}^{\mp} W_{1}^{\mp}$.

It appears that in MLRSM mixed processes, $p p \rightarrow H_{1}^{++} H_{2}^{-}$and $p p \rightarrow H_{2}^{++} H_{1}^{-}$, vanishes as $v_{L}=0$. In figure 9 the total cross section for two considered processes are given. The mass of $H_{2}^{ \pm}$is allowed to be small and because, as discussed before, this is not natural in the MLRSM, its contribution is denoted with a tilde. Anyway, its contribution (keeping a form of its couplings as dictated by MLRSM) is negligible. Final comparison of cross sections of different processes discussed in sections 2.1.1, 2.1.2 and 2.1.3 is given in 


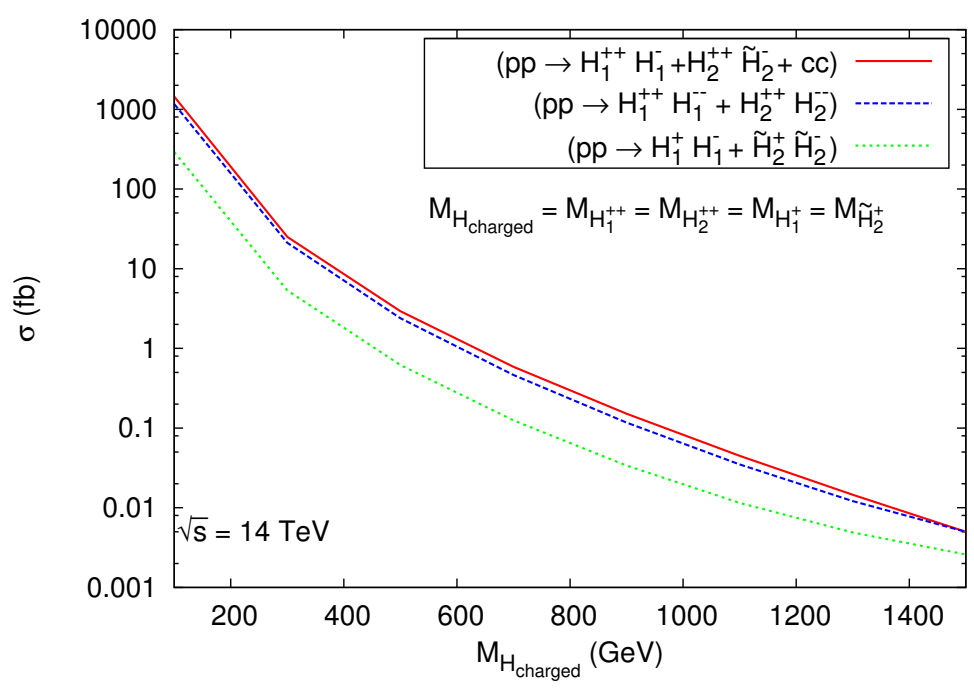

Figure 10. Summary of various MLRSM LHC production cross sections considered in the paper is shown with charged scalars at $\sqrt{s}=14 \mathrm{TeV}$ and without kinematic cuts. We have taken degenerate mass $M_{H_{\text {charged }}}$ for $M_{H_{1}^{++}}, M_{H_{2}^{++}}, M_{H_{1}^{+}}$and $M_{\tilde{H}_{2}^{+}}$.

figure 10. We can see that the largest cross sections are for a pair production of singly with doubly charged scalars, and the cross sections for production of doubly charged scalar pair is slightly lower, while the smallest cross section is for pair production of singly charged scalars. Contributions from processes where $\mathrm{H}_{2}^{ \pm}$is involved are negligible or at most much smaller than corresponding results where $H_{1}^{ \pm}$is involved. Keeping in mind the status of the SM background (analysed for our purposes in section 3.3) we look for multi lepton signals for three or more leptons. Thus we focus in the following sections on the processes which involve primary production of at least one doubly charged scalar.

\subsection{Primary production of a heavy Higgs and gauge bosons}

2.2.1 $p p \rightarrow W_{1 / 2}^{\mp} H_{1 / 2}^{ \pm}, p p \rightarrow Z_{1 / 2} H_{1 / 2}^{ \pm}$and $p p \rightarrow \gamma H_{1 / 2}^{ \pm}$

In our scenarios the production cross sections for these processes are very small and can be ignored. This is because the $W_{2} / Z_{2}$ propagator diagrams are suppressed as they are as heavy as few $\mathrm{TeV}$. For the other light propagators the scalar-gauge boson-gauge boson vertices are proportional to $\sin \xi$ and/or $v_{L}$, which are zero here.

\section{Simulations and results for final lepton signals}

In this paper we are interested in tri- and four-lepton signal events. To enhance such signals, suitable kinematic cuts are applied in order to decrease the SM backgrounds.

\subsection{Events selection criteria}

The detailed simulation criteria used in our study are following:

- The Parton Distribution Function (PDF): CTEQ6L1 [64]. 
- Initial selection (identification) criteria of a lepton: pseudorapidity $|\eta|<2.5$ and $p_{T}$ (transverse momentum $p_{T}=\sqrt{p_{x}^{2}+p_{y}^{2}}$ ) of that lepton should be $>10 \mathrm{GeV}$.

- Detector efficiency for leptons:

$\diamond$ For electron (either $e^{-}$or $e^{+}$) detector efficiency is $0.7(70 \%)$;

$\diamond$ For muon (either $\mu^{-}$or $\mu^{+}$) detector efficiency is $0.9(90 \%)$.

- Smearing of electron energy and muon $p_{T}$ are considered. All these criteria are implemented in PYTHIA and for details see [65].

- Lepton-lepton separation: the separation between any two leptons should be $\Delta R_{l l} \geq 0.2$.

- Lepton-photon separation: $\Delta R_{l \gamma} \geq 0.2$ with all the photons having $p_{T_{\gamma}}>10 \mathrm{GeV}$.

- Lepton-jet separation: the separation of a lepton with all the jets should be $\Delta R_{l j} \geq$ 0.4 , otherwise that lepton is not counted as lepton. Jets are constructed from hadrons using PYCELL within the PYTHIA.

- Hadronic activity cut: this cut is applied to take only pure kind of leptons that have very less hadronic activity around them. Each lepton should have hadronic activity, $\frac{\sum p_{T_{\text {hadron }}}}{p_{T_{l}}} \leq 0.2$ within the cone of radius 0.2 around the lepton.

- Hard $p_{T}$ cuts: $p_{T l_{1}}>30 \mathrm{GeV}, p_{T l_{2}}>30 \mathrm{GeV}, p_{T l_{3}}>20 \mathrm{GeV}, p_{T l_{4}}>20 \mathrm{GeV}$.

- Missing $p_{T}$ cut: this cut is not applied for four-lepton final states while for threelepton case due to the presence of neutrino, a missing $p_{T}$ cut $(>30 \mathrm{GeV})$ is applied.

- Z-veto ${ }^{4}$ is also applied to suppress the SM background. This has larger impact while reducing the background for four-lepton without missing energy.

\subsection{Signal events for doubly charged Higgs particles in MLRSM}

Doubly charged scalars decay mainly to either a pair of same sign charged leptons or charged gauge bosons depending on the choice of parameters. As already discussed, we have chosen the parameter space in such a way that the doubly charged scalars decay to charged leptons with almost $100 \%$ branching ratio.

This decay is lepton number violating and can also be possibly lepton flavour violating. In our scenarios we assume no lepton flavour violation as the Yukawa couplings are considered to be flavour diagonal. Thus, the four lepton final state contains two pairs of same sign and same flavoured charged leptons where each pair has opposite charges to each other. As there is no neutrino (missing energy) or jet involved it is easy to reconstruct the momentum of the final state particles. We have reconstructed invariant masses $^{5}$ for

\footnotetext{
${ }^{4}$ Same flavoured but opposite sign lepton pair invariant mass $m_{\ell_{1} \ell_{2}}$ must be sufficiently away from $Z_{1}$ mass, such that, typically, $\left|m_{\ell_{1} \ell_{2}}-M_{Z_{1}}\right| \geq 6 \Gamma_{Z_{1}} \sim 15 \mathrm{GeV}[65]$.

${ }^{5}$ The invariant mass for a lepton pair is defined as $m_{\ell_{1} \ell_{2}}=\sqrt{\left(E_{1}+E_{2}\right)^{2}-\left(\overrightarrow{P_{1}}+\overrightarrow{P_{2}}\right)^{2}}$, where $E_{i}$ and $\vec{P}_{i}$ are the energy and three momentum of $\ell_{i}$, respectively.
} 


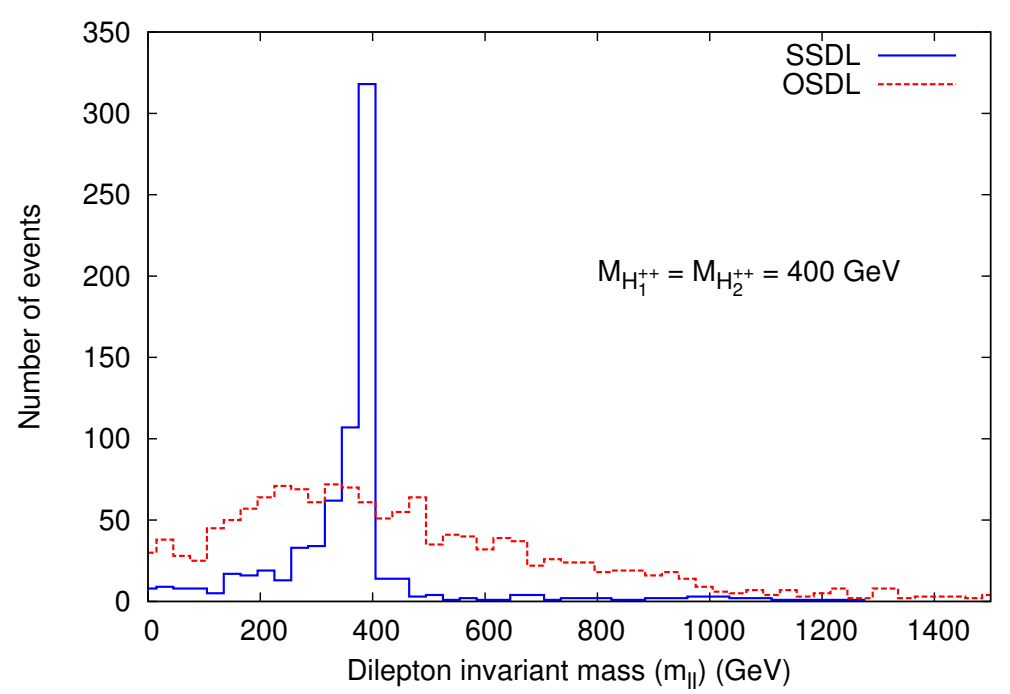

Figure 11. Invariant mass for SSDL and OSDL for $\left(p p \rightarrow H_{1,2}^{++} H_{1,2}^{--} \rightarrow 4 l\right)$ with $M_{H_{1}^{++}}=M_{H_{2}^{++}}=$ $400 \mathrm{GeV}$ for $\sqrt{s}=14 \mathrm{TeV}$ and $L=300 \mathrm{fb}^{-1}$. As the doubly charged scalars are degenerate in mass both the invariant mass peaks occur at the same place and thus cannot be distinguished.

same sign di-leptons (SSDL) and opposite sign di-leptons (OSDL). As the doubly charged scalars are the parents of the di-lepton pairs, invariant mass of the SSDL is expected to give a clean peak around the mass of the doubly charged scalar, which is not necessarily a case for OSDL.

\subsection{1 $p p \rightarrow H_{1}^{++} H_{1}^{--}$and $p p \rightarrow H_{2}^{++} H_{2}^{--}$}

Scenario I, degenerated doubly charged mass spectrum. As calculated in section 2, eq. (2.12), if $M_{H_{1}^{++}}=M_{H_{2}^{++}}=400 \mathrm{GeV}$, the cross section at the LHC with centre of mass energy $\sqrt{s}=14 \mathrm{TeV}$ is $\sigma\left(p p \rightarrow\left(H_{1}^{++} H_{1}^{--}+H_{2}^{++} H_{2}^{--}\right) \rightarrow \ell_{i}{ }^{+} \ell_{i}{ }^{+} \ell_{j}{ }^{-} \ell_{j}{ }^{-}\right)=6.06 \mathrm{fb}$, where $\ell_{i, j}=e, \mu$. After implementing all the cuts, as described in section 3.1, the four lepton events with no missing energy can be estimated. Each pair of SSDL originates from different doubly charged scalars. We have plotted the reconstructed invariant mass distributions for both SSDL and OSDL in figure 11 with anticipated integrated luminosity $L=300 \mathrm{fb}^{-1}$. As both the doubly charged scalars are degenerate the invariant mass peaks occur at around $400 \mathrm{GeV}$. This clean reconstruction of the invariant mass is indeed possible even in the hadronic environment and can be a smoking gun feature indicating the presence of doubly charged scalars.

We have computed this process also with centre of mass energy $8 \mathrm{TeV}$. In this case we find that the cross section, with $\sqrt{s}=8 \mathrm{TeV}$ at the LHC, is $1.06 \mathrm{fb}$, about 6 times smaller than for $\sqrt{s}=14 \mathrm{TeV}$. If we take present integrated luminosity to be $25 \mathrm{fb}^{-1}$ then total number of the events even before all the cuts, is statistically insignificant to analyse this particular process at the LHC after implementing all the selection criteria. Thus to justify this four lepton signal for this scenario needs more data in future.

To select the doubly charged scalar signal properly and in an independent way, there is another interesting variable which can be used for determination of signals as sug- 


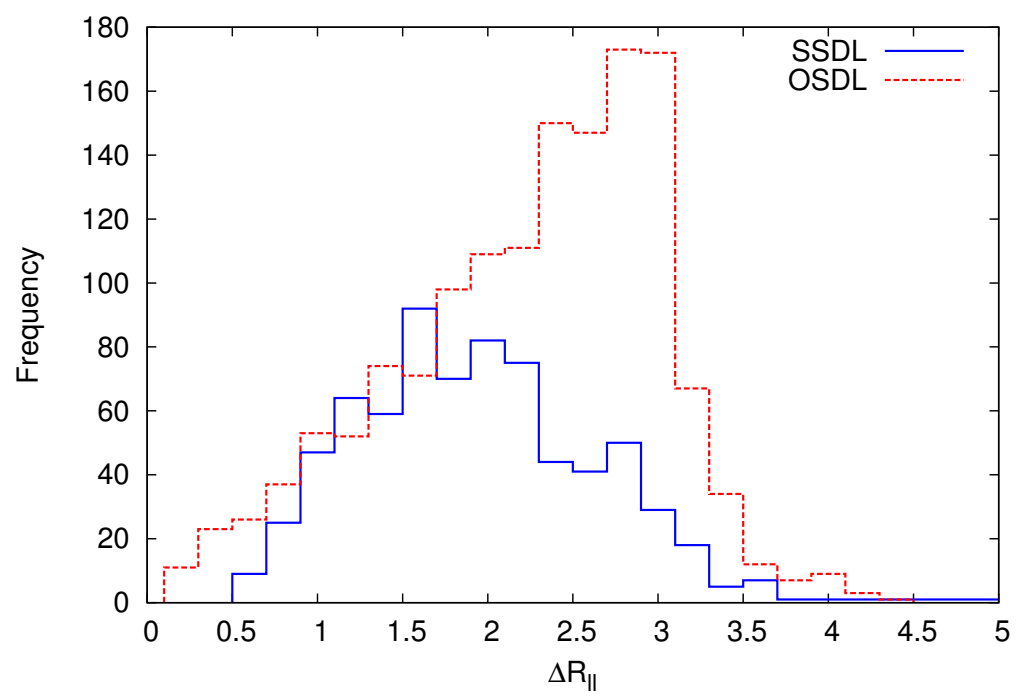

Figure 12. Lepton-lepton separations for the same sign lepton pairs $\left(\Delta R_{\ell^{ \pm} \ell^{ \pm}}\right)$and opposite sign lepton pairs $\left(\Delta R_{\ell^{ \pm} \ell^{\mp}}\right)$ for $\left(p p \rightarrow H_{1,2}^{++} H_{1,2}^{--} \rightarrow 4 l\right)$ within the degenerate scenario with $M_{H_{1}^{++}}=$ $M_{H_{2}^{++}}=400 \mathrm{GeV}$ for $\sqrt{s}=14 \mathrm{TeV}$ and $L=300 \mathrm{fb}^{-1}$.

gested in [62]

$$
\Delta R_{\ell_{1} \ell_{2}}=\sqrt{\left(\eta_{1}-\eta_{2}\right)^{2}+\left(\phi_{1}-\phi_{2}\right)^{2}}
$$

where $\eta_{i}$ and $\phi_{i}$ denote pseudorapidity and azimuth of $\ell_{i}$, respectively. $\Delta R_{\ell \ell}$ amounts the separation between two light charged leptons $(\ell)$ in azimuth-pseudorapidity plane. Its physical importance is that in the detector if $\Delta R_{\ell \ell}$ is smaller than the specified value then one can not distinguish whether the deposited energy is really by one or two leptons. So, one chooses only events for which leptons are well separated. We expect that the leptons originated from a single doubly charged scalar will be less separated than the leptons coming from different charged scalars. In our considered processes and decays the doubly charged scalars decay mainly into pair of same flavoured same sign leptons. Thus in a case of opposite sign di-lepton pair each of them are coming from different doubly charged scalars must be well separated. We have plotted the $\Delta R_{\ell \ell}$ distribution to address this feature. It is pretty clear from figure 12 that the distribution peaks at smaller $\Delta R_{\ell \ell}$ for same sign lepton pair while that for the oppositely charged lepton pair peaks at larger value of $\Delta R_{\ell \ell}$, as expected. This implies that most of the leptons in the SSDL pairs are less separated than the leptons which belong to the OSDL pair.

Scenario II, non degenerated doubly charged mass spectrum. Here we choose another set of benchmark points where the doubly charged scalars are non-degenerate. In section 2, eq. (2.18), the cross section at $\sqrt{s}=14 \mathrm{TeV}$ has been calculated for the same process with $M_{H_{1}^{ \pm \pm}}=400 \mathrm{GeV}$ and $M_{H_{2}^{ \pm \pm}}=500 \mathrm{GeV}, \sigma=4.95 \mathrm{fb}$. As $M_{H_{2}^{ \pm \pm}}>M_{H_{1}^{ \pm \pm}}$, the production cross section for $H_{1}^{ \pm \pm}$is much larger than that for $H_{2}^{ \pm \pm}$. Thus the four lepton events will be generated mostly from the leptonic decays of the $H_{1}^{ \pm \pm}$pair than $H_{2}^{ \pm \pm}$ decays. This statement is very distinctively clear from the invariant mass distributions 


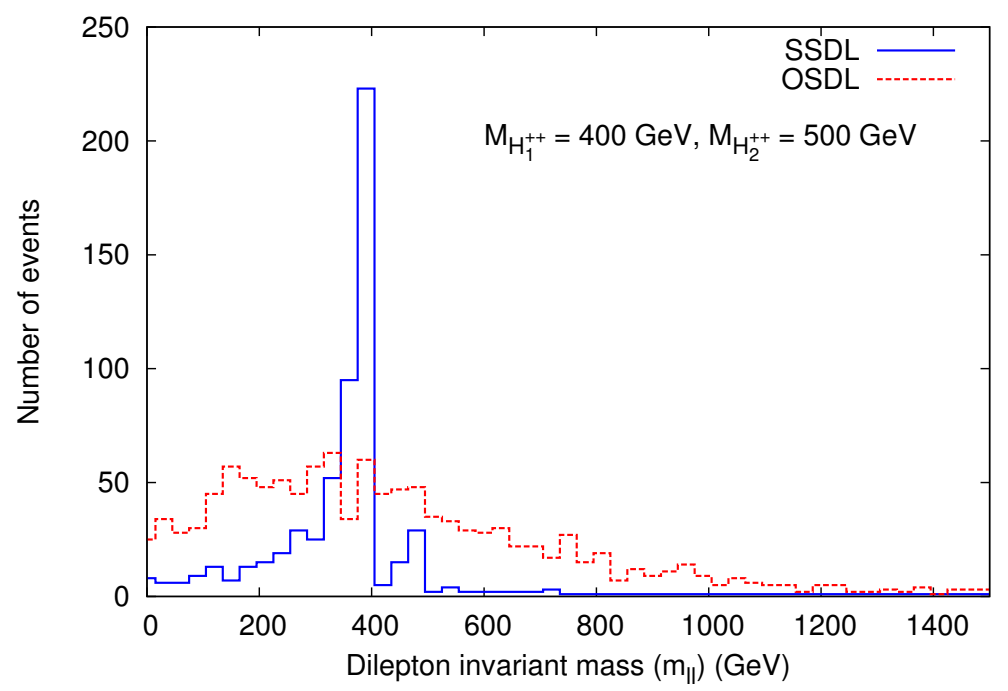

Figure 13. Invariant mass for SSDL and OSDL signals in the $\left(p p \rightarrow H_{1,2}^{++} H_{1,2}^{--} \rightarrow 4 l\right)$ process in the non-degenerate mass scenario with $M_{H_{1}^{ \pm \pm}}=400 \mathrm{GeV}$ and $M_{H_{2}^{ \pm \pm}}=500 \mathrm{GeV}$ for $\sqrt{s}=14 \mathrm{TeV}$ and $L=300 \mathrm{fb}^{-1}$.

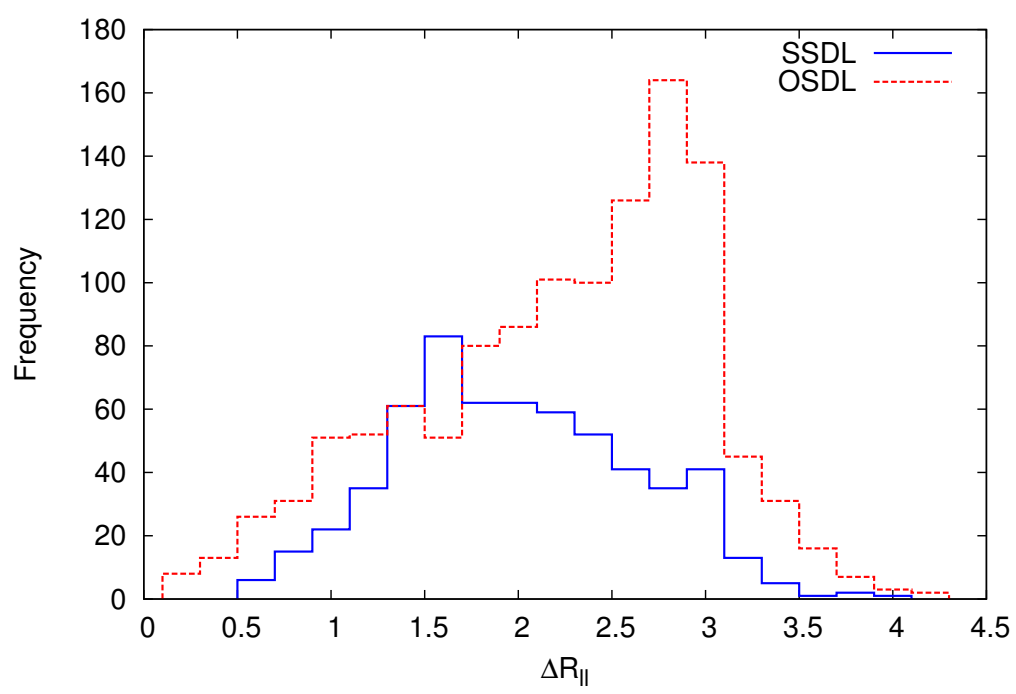

Figure 14. Lepton-lepton separations for same sign lepton pairs $\left(\Delta R_{\ell^{ \pm} \ell^{ \pm}}\right)$and opposite sign lepton pairs $\left(\Delta R_{\ell^{ \pm} \ell \mp}\right)$ in the $\left(p p \rightarrow H_{1,2}^{++} H_{1,2}^{--} \rightarrow 4 l\right)$ process for non-degenerate mass scenario having $M_{H_{1}^{ \pm \pm}}=400 \mathrm{GeV}$ and $M_{H_{2}^{ \pm \pm}}=500 \mathrm{GeV}$ with $\sqrt{s}=14 \mathrm{TeV}$ and $L=300 \mathrm{fb}^{-1}$.

of the same sign di-leptons, as shown in the figure 13. Maximum number of same dilepton events are with an invariant mass peak around $M_{H_{1}^{ \pm \pm}}=400 \mathrm{GeV}$ and that around $M_{H_{2}^{ \pm \pm}}=500 \mathrm{GeV}$ is much smaller, as expected.

We also performed the $\Delta R_{\ell \ell}$ distribution for the same benchmark point. For the same reason as explained before our expectation is reflected in figure 14 . 


\subsection{2 $p p \rightarrow H_{1}^{ \pm \pm} H_{1}^{\mp}$ and $p p \rightarrow H_{2}^{ \pm \pm} H_{2}^{\mp}$}

These processes lead to the tri-lepton events with missing $p_{T}$, see table 1 . For chosen MLRSM parameters, eq. (2.21), the cross section for the process $p p \rightarrow\left(H_{1}^{ \pm \pm} H_{1}^{\mp}+\right.$ $\left.H_{2}^{ \pm \pm} H_{2}^{\mp}\right) \rightarrow \ell \ell \ell \nu_{\ell}$ before cuts with centre of mass energy $\sqrt{s}=14 \mathrm{TeV}$ is $\sigma=6.05 \mathrm{fb}$. The tri-lepton events can be classified into two categories: either $\ell^{+} \ell^{+} \ell^{-}$or $\ell^{-} \ell^{-} \ell^{+}$. The first and second types of signals are originated from $W_{1}^{+}$and $W_{1}^{-}$mediated processes, respectively. Thus, it is indeed possible to estimate the charge asymmetry, define as the ratio of the number of events of $\ell^{+} \ell^{+} \ell^{-}$type to the number of events of $\ell^{-} \ell^{-} \ell^{+}$type at the LHC. This is very similar to the forward-backward asymmetry at Tevatron. This charge asymmetry depends on Parton Distribution Functions (PDF) and thus is a special feature of LHC. We have estimated this ratio $\left(R_{-}^{+}\right)$with the above choices of charged scalar masses with $\sqrt{s}=14 \mathrm{TeV}$ and integrated luminosity $300 \mathrm{fb}^{-1}$. We find 554 tri-lepton signal events after all the cuts and that leads to

$$
R_{-}^{+}=\frac{\# \text { of events for } \ell^{+} \ell^{+} \ell^{-}}{\# \text { of events for } \ell^{-} \ell^{-} \ell^{+}}=\frac{396}{158} \simeq 2.51
$$

In SM the corresponding value calculated for the main processes given in the next section in table 2 is $\left(R_{-}^{+}\right)_{\mathrm{SM}}=\frac{17.751}{14.962}=1.186$. This value is slightly different from the calculated values in [66] where higher order corrections are taken into account and the specific kinematic cuts are different. Nevertheless, MLRSM value given in eq. (3.2) differs substantially from its SM counterpart to signify its presence.

As discussed in section 2 , the $H_{2}^{ \pm \pm} H_{2}^{\mp} W_{1}^{\mp}$ vertex is much more suppressed compare to $H_{1}^{ \pm \pm} H_{1}^{\mp} W_{1}^{\mp}$. Thus, in this case most of the tri-lepton events are originated from $p p \rightarrow$ $H_{1}^{ \pm \pm} H_{1}^{\mp}$ process. This is clearly visible from the invariant mass distributions. Here we have plotted the same and opposite sign di-lepton invariant mass distributions, see figure 15. As similar to the earlier discussions in the opposite sign lepton pairs two leptons have different origin thus their invariant mass distribution is continuous while the same sign di-lepton invariant mass distributions always peak around the mass of the doubly charged scalars.

Here, from figure 15, it is distinctly seen that the significant amount of same sign dilepton pair peaks at $M_{H_{1}^{ \pm \pm}}=483 \mathrm{GeV}$ rather than $M_{H_{2}^{ \pm \pm}}=527 \mathrm{GeV}$. This implies that the dominant contribution to this tri-lepton events are generated through $p p \rightarrow H_{1}^{ \pm \pm} H_{1}^{\mp}$ process (cf. figure 9) and the further leptonic decays of the charged scalars.

In the figure 16, separations between leptons are plotted. As can be seen from this figure the SSDL separations peak at lower value of $\Delta R_{\ell \ell}$, while OSDL separations peak at larger value of $\Delta R_{\ell \ell}$. This is because same-sign leptons pair has the origin from the same mother, while opposite sign leptons pair has both the leptons from different mothers.

For $\sqrt{s}=8 \mathrm{TeV}$ and the same benchmark point the production cross section $\sigma(p p \rightarrow$ $\left.\left(H_{1}^{ \pm \pm} H_{1}^{\mp}+H_{2}^{ \pm \pm} H_{2}^{\mp}\right) \rightarrow \ell \ell \ell \nu_{\ell}\right)=1.44 \mathrm{fb}$ is about four times smaller than for $\sqrt{s}=14 \mathrm{TeV}$, eq. (2.21). With an integrated luminosity $25 \mathrm{fb}^{-1}$ at $\sqrt{s}=8 \mathrm{TeV}$ and $300 \mathrm{fb}^{-1}$ at $\sqrt{s}=$ $14 \mathrm{TeV}$, total number of events is about 50 times smaller in the former case, so the difference is substantial. 


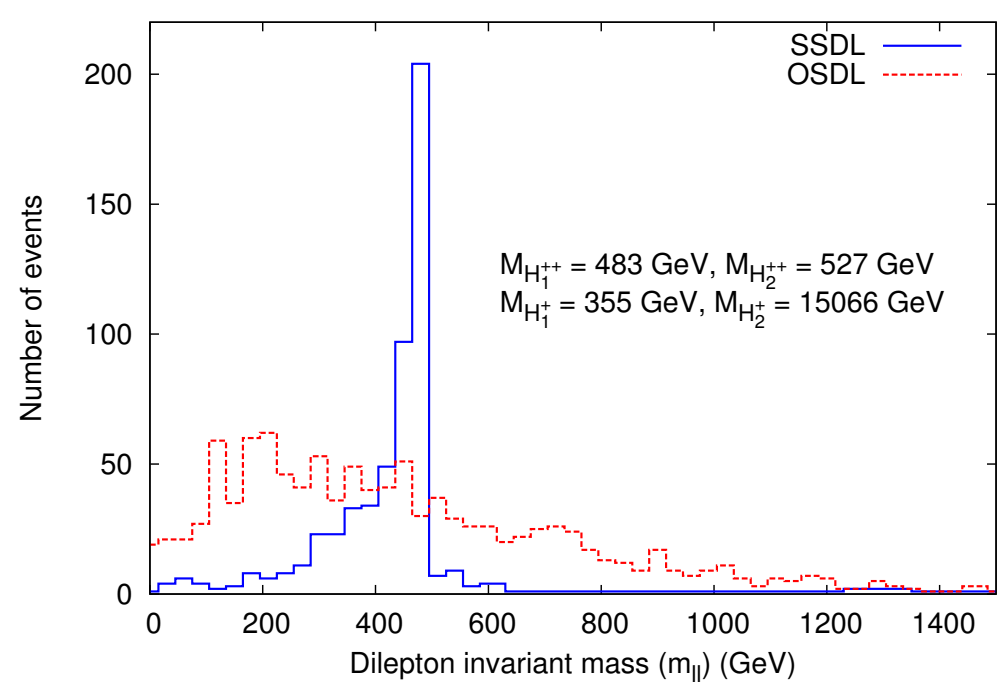

Figure 15. Invariant mass plots for SSDL and OSDL for the signals $\ell^{ \pm} \ell^{ \pm} \ell^{\mp}+$ missing $p_{T}$, at the LHC with $\sqrt{s}=14 \mathrm{TeV}$ and integrated luminosity $300 \mathrm{fb}^{-1}$.

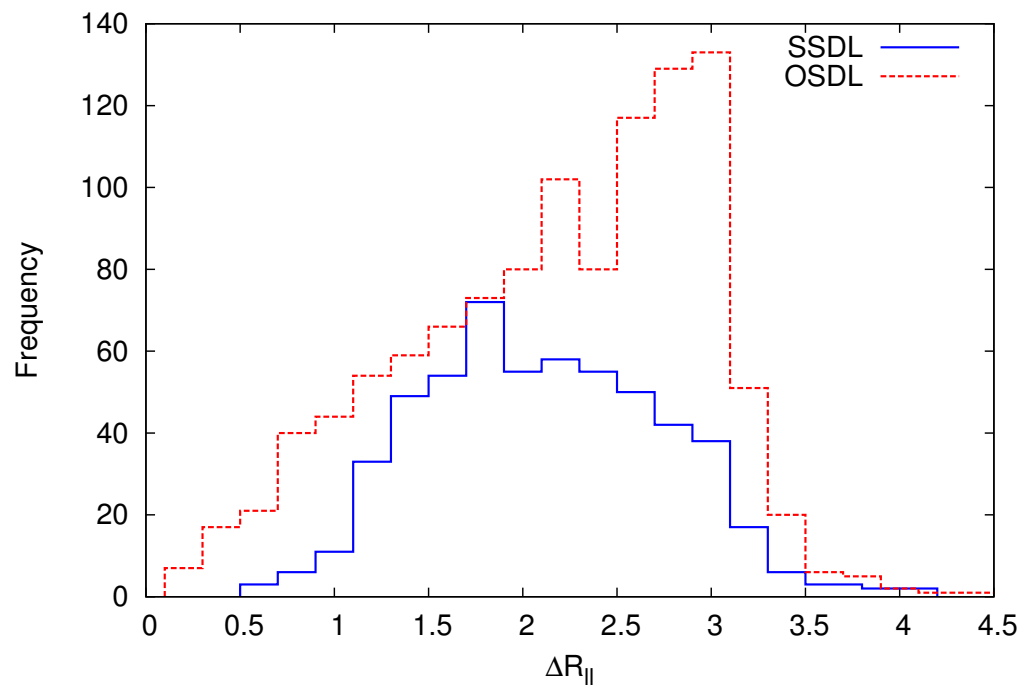

Figure 16. Lepton-lepton separation plot for same sign leptons $\left(\Delta R_{\ell^{ \pm} \ell^{ \pm}}\right)$and opposite sign leptons $\left(\Delta R_{\ell^{ \pm} \ell \mp}\right)$ in the process $\left(p p \rightarrow\left(H_{1}^{ \pm \pm} H_{1}^{\mp}+H_{2}^{ \pm \pm} H_{2}^{\mp}\right) \rightarrow 3 \ell\right)$. Here $\sqrt{s}=14 \mathrm{TeV}$ and integrated luminosity $300 \mathrm{fb}^{-1}$ at the LHC.

Distributions presented so far show that it is possible to extract clear signals for doubly charged scalars at the LHC. However, for signal identification crucial is how large the SM background effects are and the significance too.

\subsection{Background estimation and significance of signals}

Kinematic cuts are used which have been investigated and established for the first time in [65]. The cuts are optimised in a way such that we can reduce the SM background 


\begin{tabular}{|c|c|c|c|c|}
\hline Processes & $3 \ell(\mathrm{fb})$ & $\ell^{+} \ell^{+} \ell^{-}(\mathrm{fb})$ & $\ell^{-} \ell^{-} \ell^{+}(\mathrm{fb})$ & $4 \ell(\mathrm{fb})$ \\
\hline$t \bar{t}$ & 18.973 & 9.522 & 9.451 & - \\
\hline$t \bar{t}\left(Z / \gamma^{\star}\right)$ & 1.103 & 0.549 & 0.552 & 0.0816 \\
\hline$t \bar{t} W^{ \pm}$ & 0.639 & 0.422 & 0.214 & - \\
\hline$W^{ \pm}\left(Z / \gamma^{\star}\right)$ & 10.832 & 6.664 & 4.164 & - \\
\hline$\left(Z / \gamma^{\star}\right)\left(Z / \gamma^{\star}\right)$ & 1.175 & 0.594 & 0.581 & 0.0362 \\
\hline \hline TOTAL & 32.722 & 17.751 & 14.962 & 0.1178 \\
\hline
\end{tabular}

Table 2. Dominant Standard Model background contributions (in $\mathrm{fb}$ ) for tri- and four-lepton signals at the LHC with $\sqrt{s}=14 \mathrm{TeV}$ after obeying suitable selection criteria defined in the text. The $t \bar{t}$ cross section is presented here after the inclusion of k-factor. While computing the SM contributions to $4 \ell$ final state, no missing $p_{T}$ cut has been applied.

and enhance the signal events. ${ }^{6}$ Standard Model background cross sections for tri- and four-lepton signals are given in table 2 . In this table we have also separately computed the backgrounds for $\ell^{+} \ell^{+} \ell^{-}$and $\ell^{-} \ell^{-} \ell^{+}$.

In principal the tri-lepton contributions can come also from $\mathrm{H}_{1}^{++} \mathrm{H}_{1}^{--}$and $\mathrm{H}_{2}^{++} \mathrm{H}_{2}^{--}$ involved processes if during simulations one of the four-leptons does not satisfy the cuts. But in our case this contribution is negligible due to the extra missing energy cut applied as one of the gate pass for the tri-lepton events. Thus all the productions together are considered and all the intermediate particles are allowed to decay. After passing through the cuts, tri-lepton and four-lepton events are counted.

In table 3 we present the total background and signal events for 25 and $300 \mathrm{fb}^{-1}$ integrated luminosities. It is clear that four-lepton signals are well beyond the SM background. The tri-lepton signal is also very prominent over the background (what matters is the signal excess over the background fluctuations). To see it properly, in table 4 the significance of different signals is shown.

Assuming the significance at the level of 5 as a comfortable discovery limit, we can see that LHC will be in the next run sensitive to masses of MLRSM doubly charged Higgs bosons up to approximately $600 \mathrm{GeV}$.

\section{MLRSM charged Higgs bosons contribution to $\boldsymbol{H}_{0}^{0} \rightarrow \gamma \gamma$}

In LR symmetric models there are (singly-, doubly-) charged scalars and charged gauge boson $\left(W_{2}^{ \pm}\right)$which couple to photons and hence they can contribute to $H_{0}^{0} \rightarrow \gamma \gamma$ channel where $H_{0}^{0}$ is the SM-like neutral Higgs taken to be $125 \mathrm{GeV}$. Since $W_{2}^{ \pm}$are heavy, their

\footnotetext{
${ }^{6}$ In our analysis while computing the tri-lepton events (signal and background), the $p_{T}$ of the third hardest lepton needs to be greater than $20 \mathrm{GeV}$, and also a missing $p_{T}$ cut $(>30 \mathrm{GeV})$ must be satisfied, see section 3.1. Thus the tri-lepton background for process like $t \bar{t}$ where one of the lepton is coming from semi-leptonic decays of $B$ 's is reduced. Here the hadronic activity cut also reduces the hadronic activity around the selected leptons and plays a crucial role in this case. All these cuts reduces the efficiency of misidentification of b-jets as leptons. In our case this is less than $0.05 \%$.
} 


\begin{tabular}{|c|c|c|c|c|c|}
\hline \multirow{2}{*}{ Luminosity } & Background & Signal & Background & \multicolumn{2}{|c|}{ Signal $4 \ell$ events } \\
\hline & $3 \ell$ events & $3 \ell$ events & $4 \ell$ events & scenario I & scenario II \\
\hline $25 \mathrm{fb}^{-1}$ & 797.5 & 46.2 & 2.9 & $\begin{array}{l}\text { (i) } 30 \\
\text { (ii) } 4.4\end{array}$ & 24.8 \\
\hline $300 \mathrm{fb}^{-1}$ & 9569.7 & 554 & 34.8 & $\begin{array}{l}\text { (i) } 360 \\
\text { (ii) } 53\end{array}$ & 298 \\
\hline
\end{tabular}

Table 3. Number of background and signal events at $25 \mathrm{fb}^{-1}$ and $300 \mathrm{fb}^{-1}$ as an anticipated integrated luminosity at next $14 \mathrm{TeV}$ run of LHC. The tri-lepton signal is computed for following charged scalar masses: $M_{H_{1}^{ \pm \pm}}=483 \mathrm{GeV}, M_{H_{2}^{ \pm \pm}}=527 \mathrm{GeV}, M_{H_{1}^{ \pm}}=355 \mathrm{GeV}, M_{H_{2}^{ \pm}}=15066 \mathrm{GeV}$. Scenario I reflects degeneracy of doubly charged scalar masses with (i) $M_{H_{1}^{ \pm \pm}}=M_{H_{2}^{ \pm \pm}}=400 \mathrm{GeV}$ and (ii) $M_{H_{1}^{ \pm \pm}}=M_{H_{2}^{ \pm \pm}}=600 \mathrm{GeV}$, while Scenario II realises their non-degenerate spectrum, namely $M_{H_{1}^{ \pm \pm}}=400$ and $M_{H_{2}^{ \pm \pm}}=500 \mathrm{GeV}$. Here we have used the same kinematical cuts as applied while estimating the SM background events. We have not implemented other extra cuts, like invariant mass $\left(m_{\ell \ell}\right)$ and lepton separation $\left(\Delta R_{\ell \ell}\right)$ to estimate the signal and background events in this table.

\begin{tabular}{|c|c|c|c|}
\hline Significance & $3 \ell$ events & \multicolumn{2}{|c|}{$4 \ell$ events } \\
& & scenario I & scenario II \\
\hline$S / \sqrt{B}$ & 5.66 & $\begin{array}{c}\text { (i) NA } \\
\text { (ii) NA }\end{array}$ & NA \\
\hline$S / \sqrt{(S+B)}$ & 5.51 & $\begin{array}{c}\text { (i) } 18.11 \\
\text { (ii) } 5.65\end{array}$ & 16.34 \\
\hline
\end{tabular}

Table 4. The significance of the signals given in table 3 is given using two definitions of significance: (i) $S / \sqrt{B}$, and (ii) $S / \sqrt{(S+B)}$, where $S$ and $B$ are the total number of signal and background events for $300 \mathrm{fb}^{-1}$ integrated luminosity, respectively. The parameters are the same as given in table 3 . Here 'NA' implies that $S / \sqrt{B}$ can not be used as the definition of significance in these cases as $S \ll B$ is not justified.

contributions are suppressed compared to charged scalars, so we look for charged scalar contributions. They contribute to the channel via a loop shown in the figure 17 .

Following [67-69] we can write the enhancement factor for this channel, which is nothing but a ratio of partial decay width in the new model to that in the SM

$$
R_{\gamma \gamma}=\left|1+\sum_{S=H_{1,2}^{ \pm \pm}, H_{1}^{ \pm}} Q_{S}^{2} \frac{c_{S}}{2} \frac{k_{+}^{2}}{M_{S}^{2}} \frac{A_{0}\left(\tau_{S}\right)}{A_{1}\left(\tau_{W_{1}}\right)+N_{c} Q_{t}^{2} A_{1 / 2}\left(\tau_{t}\right)}\right|^{2}
$$

In the above equation $Q_{S}$ is electric charge of charged scalars in unit of $e, M_{S}$ is a mass of scalars. $N_{c}$ is colour factor which is 1 for colour singlet scalars and $\tau_{i}=4 m_{i}^{2} / m_{H_{0}^{0}}^{2}$ $\left(i=W_{1}, t, S\right) . c_{S}$ are the coupling of the Higgs boson with the charged scalars and $k_{+}=$ $\sqrt{k_{1}^{2}+k_{2}^{2}}$ where $k_{1}, k_{2}$ are the vacuum expectation values of the bi-doublet. The expressions 


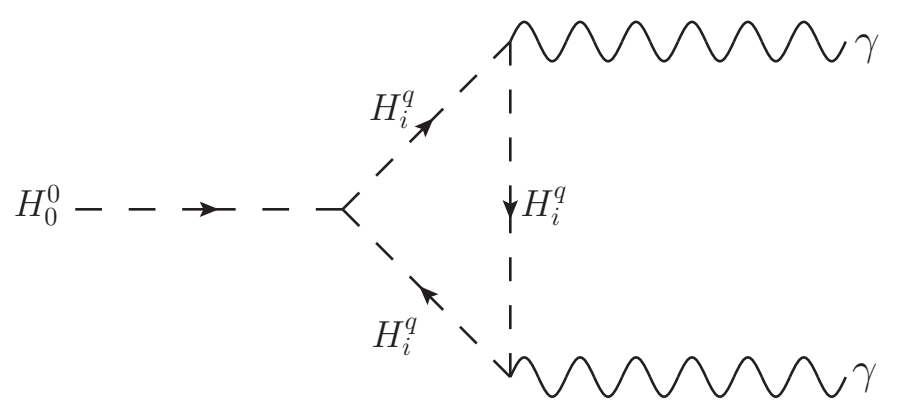

Figure 17. Charged scalar contribution to the $H_{0}^{0} \rightarrow \gamma \gamma$ channel at the LHC. In the loop there are three contributions coming from the charged scalars $H_{i}^{q} \equiv H_{1}^{ \pm \pm}, H_{2}^{ \pm \pm}, H_{1}^{ \pm}$. In MLRSM $H_{2}^{ \pm}$is very heavy and its contribution is negligible.

for $c_{S}$ are as follows

$$
\begin{aligned}
c_{H_{0}^{0} H_{1}^{+} H_{1}^{-}} & =-\left[\frac{2 \alpha_{1} k_{+}^{2}+8 \alpha_{2} k_{1} k_{2}+\alpha_{3}\left(k_{+}^{2}\right)}{2 k_{+}^{2}}\right], \\
c_{H_{0}^{0} H_{1}^{++} H_{1}^{--}} & =-\left[\frac{\alpha_{1} k_{+}^{2}+k_{1}\left(4 \alpha_{2} k_{2}+\alpha_{3} k_{1}\right)}{k_{+}^{2}}\right], \\
c_{H_{0}^{0} H_{2}^{++} H_{2}^{--}} & =-\left[\frac{\alpha_{1} k_{+}^{2}+k_{1}\left(4 \alpha_{2} k_{2}+\alpha_{3} k_{1}\right)}{k_{+}^{2}}\right] .
\end{aligned}
$$

Here the parameters that are involved in the above eqs. (4.2)-(4.4), are contained in the scalar potential and following the convention as suggested in [36].

$A_{1 / 2}, A_{1}$ and $A_{0}$ are loop functions for fermions, vector bosons and scalars respectively, given as

$$
\begin{aligned}
A_{1 / 2}(x) & =2 x^{2}\left[x^{-1}+\left(x^{-1}-1\right) f\left(x^{-1}\right)\right], \\
A_{1}(x) & =-x^{2}\left[2 x^{-2}+3 x^{-1}+3\left(2 x^{-1}-1\right) f\left(x^{-1}\right)\right], \\
A_{0}(x) & =-x^{2}\left[x^{-1}-f\left(x^{-1}\right)\right] .
\end{aligned}
$$

For the SM-like Higgs mass below threshold, i.e., $m_{H_{0}^{0}}<2 m_{\text {loop }}\left(m_{\text {loop }}\right.$ is a mass of a particle in the loop) $f(x)=\operatorname{arcSin}^{2}(\sqrt{x})$.

In figure 18 we present a contour plot to grab the contributions from the charged scalars to $R_{\gamma \gamma}$. We have assumed $M_{H_{1}^{++}}=M_{H_{1}^{+}}$to reduce number of free parameters.

Experimental observations of the Higgs to di-photon decay normalised to the SM prediction, as pointed out by ATLAS and CMS is given as in [70, 71]:

$$
\begin{aligned}
& R_{\gamma \gamma}=1.65 \pm 0.24 \text { (stat) }{ }_{-0.18}^{+0.25} \text { (syst) (ATLAS), } \\
& R_{\gamma \gamma}=0.78_{-0.26}^{+0.28} \quad(\mathrm{CMS}) .
\end{aligned}
$$

As errors are still very large, it is too early to make any conclusive remarks on these results, especially that tendency seems to be that anomaly systematically approaches 1 . However, MLRSM can accommodate wide range of $R_{\gamma \gamma}$ values by the charged Higgs boson effects, for related discussions, see e.g. in [72]. 


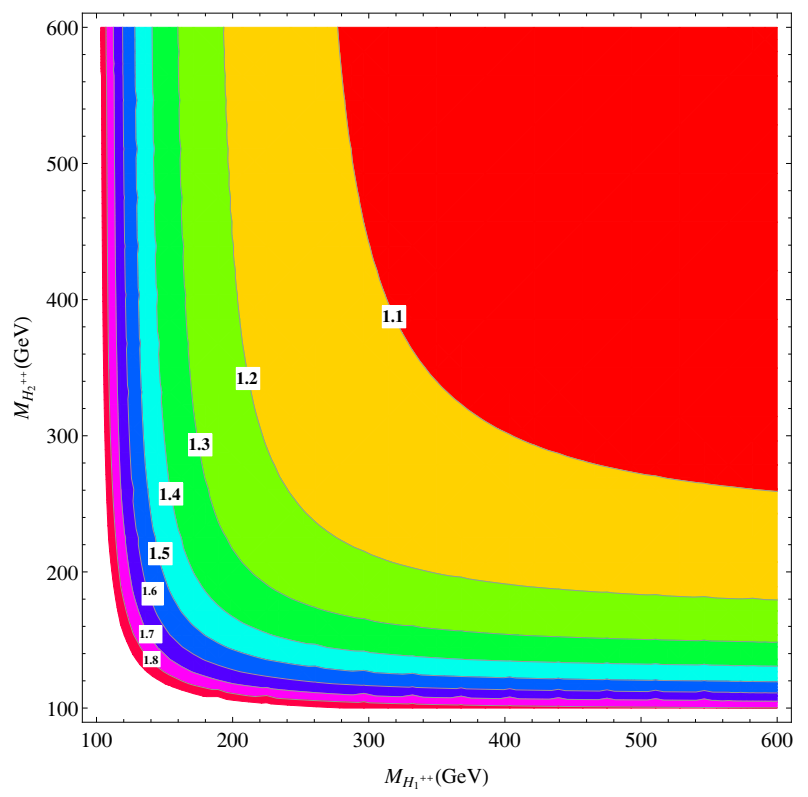

Figure 18. $R_{\gamma \gamma}$ with the variation of charged scalar masses.

\section{Conclusions and outlook}

After discovery of the SM-like neutral Higgs boson in July 2012 at the LHC, the next big issue is what is the actual shape of the Higgs potential. Thus a question is asked to reveal the further query regarding possible gauge symmetry behind the existence of elementary Higgs boson. Here we have concentrated on studies connected with LHC potential discovery of charged Higgs bosons within classical MLRSM which is already phenomenologically rich enough and worth of separate investigations. Though different low energy data and the LHC exclusion plots constrain already $W_{2}$ and $Z_{2}$ very much, still the charged scalars can be relatively light. It has been shown which of singly and doubly charged Higgs bosons can be light, in agreement with FCNC limits on neutral Higgs bosons particles, as both charged and neutral scalar sectors are connected through the Higgs potential parameters. They can be produced at the LHC with non-negligible cross sections. However, their production cross sections decrease rapidly with their masses, that is why we have undertaken here more detailed and systematic studies including the production and decays of charged scalars. We have concentrated on the single and pair production of doubly charged scalars. We have chosen the benchmark points in such a way that signals connected with doubly charged scalars can dominate over non-standard signals coming from both heavy gauge and neutral Higgs bosons. We have analysed the four-lepton and tri-lepton signals at the LHC. As a rule of thumb, for all considered processes with doubly charged scalars cross sections are about $1 \mathrm{fb}$ for their masses in vicinity of $400 \div 500 \mathrm{GeV}$, which is about the present lowest limit on their masses. If planed integrated luminosity in the next LHC run at $\sqrt{s}=14 \mathrm{TeV}$ is about 10 times larger than present values, clear signals with four-leptons without missing energy and tri-lepton signals can be detected. It will be an indication 
for doubly charged scalar effects. These multi lepton final states posses very small SM background. We have shown that MLRSM model can give such signals for doubly charged masses up to approximately $600 \mathrm{GeV}$. In our analysis we have used the di-lepton invariant mass and lepton-lepton separation distributions. We also estimate the amount of charge asymmetry in signal as well as background events, and show that this might be a smoking gun feature for future discovery. The same and opposite sign charged lepton signals have been analysed using proper kinematic cuts and the clear impact of doubly charged scalars are noted carefully.

Finally, as in the Left-Right symmetric models charged gauge bosons are very heavy, they do not contribute significantly to the Higgs to di-photon process, however, the relatively light charged scalars can contribute easily. We have incorporated the impact of the light charged scalars in this process and estimated the strength of this contribution over the SM one.

As an outlook, several interesting things can still be done, e.g.

1. More detailed comparison studies including also lepton spin correlations and their angular distributions with other non-standard models where doubly charged scalars exist (e.g. Higgs Triplet Model [73]);

2. Studies of dedicated distributions for processes involving doubly charged Higgs bosons with both jets and missing energy;

3. Theoretical studies of general Higgs potentials which can realise relatively light charged Higgs bosons keeping at the same time a few $\mathrm{TeV}$ scale of neutral Higgs bosons (e.g. [48]);

4. To release theoretical assumptions on equality of left and right gauge boson couplings, diagonal neutrino light-heavy mixings and possible see-saw scenarios, take into account relations between model parameters in the Higgs, gauge and neutrino sectors, e.g. [11].

In summary, we are in a very exciting moment and the next LHC run should be decisive if our scenario with relatively light charged Higgs bosons can be realised. Still there is a room for Left-Right gauge symmetry signals discovery at the LHC, including MLRSM doubly charged Higgs bosons effects as long as long as their masses will be well below $1 \mathrm{TeV}$ range $\left(m_{H_{1 / 2}^{ \pm \pm}} \leq 600 \mathrm{GeV}\right)$.

\section{Acknowledgments}

Work of JC is supported by Department of Science \& Technology, Government of INDIA under the Grant Agreement number IFA12-PH-34 (INSPIRE Faculty Award). Work of MK is supported by the Świder fellowship. Work of JG is supported by the Research Executive Agency (REA) of the European Union under the Grant Agreement number PITN-GA-2010264564 (LHCPhenoNet). Work of RS is supported by Science and Engineering Research Canada (NSERC). 


\section{A Reconciling FCNC effects and large $v_{R}$ with relatively light charged Higgs mass spectrum within MLRSM}

A scan of potential parameters based on the numerical diagonalisation and minimisation of the complete MLRSM Higgs potential within our own implementation of the FeynRules package [46] has been performed. This leads to the figure 1. Here, just for illustration, we discuss it in a simplified form based on approximations discussed in [74]. In MLRSM there is one neutral SM-like Higgs boson having mass proportional to the vacuum expectation value $(\mathrm{VEV}) \kappa_{1}$ ( electro-weak breaking scale). The other Higgs bosons are much heavier. A natural mass scale for them is driven by $v_{R}$ which decides about the $\mathrm{SU}(2)_{R} \otimes \mathrm{U}(1)_{B-L}$ breaking scale. As discussed in the main text of the paper, we assume large $v_{R}(\sim 8 \mathrm{TeV})$, to be consistent with the experimental constraints.

The minimisation and diagonalisation of the MLRSM Higgs potential have been investigated in [35] and explicit correlations among physical and unphysical scalar fields are given in [74]. For the sake of completeness, here we have depicted them along with their mass relations considering $\kappa_{2}=0$ :

- masses

$$
\begin{array}{rlrl}
M_{H_{0}^{0}}^{2} & \simeq 2 \kappa_{1}^{2} \lambda_{1}, & & \\
M_{H_{1}^{0}}^{2} & \simeq \frac{1}{2} \alpha_{3} v_{R}^{2}, & & M_{H_{3}^{0}}^{2} \simeq \frac{1}{2} v_{R}^{2}\left(\rho_{3}-2 \rho_{1}\right), \\
M_{H_{2}^{0}}^{2} & \simeq 2 \rho_{1} v_{R}^{2}, & & \\
M_{A_{1}^{0}}^{2} & \simeq \frac{1}{2} \alpha_{3} v_{R}^{2}-2 \kappa_{1}^{2}\left(2 \lambda_{2}-\lambda_{3}\right), & & \\
M_{A_{2}^{0}}^{2} & \simeq \frac{1}{2} v_{R}^{2}\left(\rho_{3}-2 \rho_{1}\right), \\
M_{H_{1}^{ \pm}}^{2} & \simeq \frac{1}{2} v_{R}^{2}\left(\rho_{3}-2 \rho_{1}\right)+\frac{1}{4} \alpha_{3} \kappa_{1}^{2}, & M_{H_{2}^{ \pm}}^{2} & \simeq \frac{1}{2} \alpha_{3}\left[v_{R}^{2}+\frac{1}{2} \kappa_{1}^{2}\right], \\
M_{H_{1}^{ \pm \pm}}^{2} & \simeq \frac{1}{2}\left[v_{R}^{2}\left(\rho_{3}-2 \rho_{1}\right)+\alpha_{3} \kappa_{1}^{2}\right], & M_{H_{2}^{ \pm \pm}}^{2} & \simeq 2 \rho_{2} v_{R}^{2}+\frac{1}{2} \alpha_{3} \kappa_{1}^{2} .
\end{array}
$$

- relations among physical and unphysical fields ("G" stands for Goldstone modes)

$$
\begin{array}{rlrl}
\phi_{1}^{0} & \simeq \frac{1}{\sqrt{2}}\left[H_{0}^{0}+i \tilde{G}_{1}^{0}\right], & & \\
\phi_{2}^{0} & \simeq \frac{1}{\sqrt{2}}\left[H_{1}^{0}-i A_{1}^{0}\right], & & \\
\delta_{R}^{0} & =\frac{1}{\sqrt{2}}\left(H_{2}^{0}+i G_{2}^{0}\right), & \delta_{L}^{0} & =\frac{1}{\sqrt{2}}\left(H_{3}^{0}+i A_{2}^{0}\right), \\
\delta_{L}^{+} & =H_{1}^{+}, & \delta_{R}^{+} & \simeq G_{R}^{+}, \\
\phi_{1}^{+} & \simeq H_{2}^{+}, & \phi_{2}^{+} \simeq G_{L}^{+} \\
\delta_{R}^{ \pm \pm} & =H_{1}^{ \pm \pm}, & \delta_{L}^{ \pm \pm} & =H_{2}^{ \pm \pm} .
\end{array}
$$


As masses of quarks are non-degenerate, FCNC effects appear through the $A_{0}$ part of the following Lagrangian [36]

$$
\begin{aligned}
L_{\text {quark-Higgs }}(u, d)=-\bar{U}[ & P_{L}\left(M_{\text {diag }}^{u} B_{0}^{*}+U^{\mathrm{CKM}} M_{\text {diag }}^{d} U^{\mathrm{CKM} \dagger} A_{0}\right) \\
& \left.+P_{R}\left(M_{\text {diag }}^{u} B_{0}+U^{\mathrm{CKM}} M_{\text {diag }}^{d} U^{\mathrm{CKM} \dagger} A_{0}^{*}\right)\right] U,
\end{aligned}
$$

where $A_{0}$ is a linear combination of neutral physical Higgs and Goldstone fields connected with a bi-doublet $\Phi$ [35], and taking into account eq. (A.9), we finally have

$$
A_{0}=\sqrt{2}\left(\kappa_{1} \phi_{2}^{0}\right)=\left(H_{1}^{0}-i A_{1}^{0}\right) .
$$

To suppress the effects connected with these fields [7, 28, 75-77], their masses needs to be at least $\sim 10 \mathrm{TeV}$. In our analysis we have kept them to be $\sim 15 \mathrm{TeV}$ :

$$
m_{H_{1}^{0}}, m_{A_{1}^{0}}>15 \mathrm{TeV} .
$$

It can be easily shown that for defined masses of Higgs bosons, see eqs. (A.2)-(A.7), we can find parameters of the MLRSM Higgs potential within the perturbative limit, and simultaneously satisfy the light charged Higgs bosons and eq. (A.16). This can be achieved even after keeping three charged Higgs bosons $H_{1}^{ \pm \pm}, H_{2}^{ \pm \pm}, H_{1}^{ \pm}$relatively light. For instance, with $v_{R}=8 \mathrm{TeV}$ and $\kappa_{1}=246 \mathrm{GeV}$ we find the scalar spectrum (in GeV)

$$
\begin{aligned}
M_{H_{0}^{0}} & =125, & & \\
M_{H_{1}^{0}} & =15062, & & M_{H_{2}^{0}}=11313, \\
M_{A_{1}^{0}} & =15066, & & \\
M_{A_{2}^{0}} & =505, & & \\
M_{H_{1}^{ \pm}} & =602, & & \\
M_{H_{1}^{ \pm \pm}} & =685, & M_{H_{2}^{ \pm}}=1505, & M_{H_{2}^{ \pm \pm}}=463,
\end{aligned}
$$

where

$$
\begin{array}{lll}
\rho_{1}=1, & \rho_{2}=0, & \rho_{3}=2.008, \\
\lambda_{1}=0.13, & \lambda_{2}=0, & \lambda_{3}=1, \\
\alpha_{3}=7.09 . & &
\end{array}
$$

We can see that the remaining fourth charged Higgs boson $H_{2}^{ \pm}$in MLRSM is naturally very heavy. To make it light, one needs to go beyond MLRSM and incorporate new terms in the scalar potential which would affect MLRSM Higgs boson masses. ${ }^{7}$

\footnotetext{
${ }^{7}$ Let us imagine that an additional intermediate energy scale is introduced connected with VEV of an additional $\mathrm{SU}(2)_{L}$ and $\mathrm{SU}(2)_{R}$ singlet scalar field (such scalars give for instance heavy neutrino Majorana mass terms but they decouple from other low energy phenomenological effects). If this scalar couple to the MLRSM right handed triplet fields, it would modify eqs. (A.6), (A.7) but because of its large VEV, mixing of MLRSM Higgs scalars with this state would be negligible, so the effective couplings of MLRSM Higgs bosons, including $H_{2}^{ \pm}$, would stay the same.
} 
Open Access. This article is distributed under the terms of the Creative Commons Attribution License (CC-BY 4.0), which permits any use, distribution and reproduction in any medium, provided the original author(s) and source are credited.

\section{References}

[1] R.N. Mohapatra and J.C. Pati, A natural left-right symmetry, Phys. Rev. D 11 (1975) 2558 [INSPIRE].

[2] G. Senjanović and R.N. Mohapatra, Exact left-right symmetry and spontaneous violation of parity, Phys. Rev. D 12 (1975) 1502 [InSPIRE].

[3] M. Nemevšek, F. Nesti, G. Senjanović and Y. Zhang, First limits on left-right symmetry scale from LHC data, Phys. Rev. D 83 (2011) 115014 [arXiv:1103.1627] [INSPIRE].

[4] A. Ferrari et al., Sensitivity study for new gauge bosons and right-handed Majorana neutrinos in pp collisions at $\sqrt{s}=14$ TeV, Phys. Rev. D 62 (2000) 013001 [INSPIRE].

[5] M. Frank, A. Hayreter and I. Turan, Top quark pair production and asymmetry at the Tevatron and LHC in left-right models, Phys. Rev. D 84 (2011) 114007 [arXiv:1108.0998] [INSPIRE].

[6] S.P. Das, F.F. Deppisch, O. Kittel and J.W.F. Valle, Heavy neutrinos and lepton flavour violation in left-right symmetric models at the LHC, Phys. Rev. D 86 (2012) 055006 [arXiv: 1206.0256] [INSPIRE].

[7] J. Chakrabortty, J. Gluza, R. Sevillano and R. Szafron, Left-right symmetry at LHC and precise 1-loop low energy data, JHEP 07 (2012) 038 [arXiv:1204.0736] [INSPIRE].

[8] C.-H. Lee, P.S. Bhupal Dev and R.N. Mohapatra, Natural TeV-scale left-right seesaw for neutrinos and experimental tests, Phys. Rev. D 88 (2013) 093010 [arXiv:1309.0774] [INSPIRE].

[9] X.-G. He and G. Valencia, B decays with $\tau$-leptons in non-universal left-right models, Phys. Rev. D 87 (2013) 014014 [arXiv: 1211.0348] [InSPIRE].

[10] Particle Data Group collaboration, J. Beringer et al., Review of particle physics, Phys. Rev. D 86 (2012) 010001 [InSPIRE].

[11] M. Czakon, J. Gluza and M. Zralek, Low-energy physics and left-right symmetry: bounds on the model parameters, Phys. Lett. B 458 (1999) 355 [hep-ph/9904216] [INSPIRE].

[12] CMS collaboration, Search for narrow resonances using the dijet mass spectrum with 19.6 $\mathrm{fb}^{-1}$ of $p p$ collisions at $\sqrt{\mathrm{s}}=8 \mathrm{TeV}$, CMS-PAS-EXO-12-059 (2013).

[13] CMS collaboration, Search for resonances in the dilepton mass distribution in pp collisions at $\sqrt{s}=8 \mathrm{TeV}$, CMS-PAS-EXO-12-061 (2013).

[14] ATLAS collaboration, Search for new phenomena in the dijet mass distribution updated using $13.0 \mathrm{fb}^{-1}$ of $\mathrm{pp}$ collisions at sqrts $=8 \mathrm{TeV}$ collected by the ATLAS detector, ATLAS-CONF-2012-148, ATLAS-COM-CONF-2012-180 (2012).

[15] ATLAS collaboration, Search for $W^{\prime} \rightarrow t \bar{b}$ in proton-proton collisions at a centre-of-mass energy of $\sqrt{s}=8 \mathrm{TeV}$ with the ATLAS detector, ATLAS-CONF-2013-050,

ATLAS-COM-CONF-2013-022 (2013). 
[16] ATLAS collaboration, Search for high-mass dilepton resonances in $20 \mathrm{fb}^{-1}$ of $\mathrm{pp}$ collisions at $\sqrt{s}=8 \mathrm{TeV}$ with the ATLAS experiment, ATLAS-CONF-2013-017,

ATLAS-COM-CONF-2013-010 (2013).

[17] CMS collaboration, Search for a heavy neutrino and right-handed $W$ of the left-right symmetric model in pp collisions at 8 TeV, CMS-PAS-EXO-12-017 (2012).

[18] CMS collaboration, Searches for new physics with leptons and/or jets at CMS, CMS-CR-2013-155 (2013) [arXiv: 1307.2168] [inSPIRE].

[19] LEP Higgs Working Group for Higgs boson searches, ALEPH, DELPHI, L3 and OPAL collaborations, Search for charged Higgs bosons: preliminary combined results using LEP data collected at energies up to $209 \mathrm{GeV}$, hep-ex/0107031 [INSPIRE].

[20] CMS collaboration, Search for a light charged Higgs boson in top quark decays in pp collisions at $\sqrt{s}=7 \mathrm{TeV}$, JHEP 07 (2012) 143 [arXiv: 1205. 5736] [INSPIRE].

[21] ATLAS collaboration, Search for charged Higgs bosons in the $\tau+$ jets final state with $p p$ collision data recorded at $\sqrt{s}=8 \mathrm{TeV}$ with the ATLAS experiment, ATLAS-CONF-2013-090 (2013).

[22] CMS collaboration, Inclusive search for doubly charged Higgs in leptonic final states with the 2011 data at $7 \mathrm{TeV}$, CMS-PAS-HIG-12-005 (2012).

[23] ATLAS collaboration, Search for doubly-charged Higgs bosons in like-sign dilepton final states at $\sqrt{s}=7 \mathrm{TeV}$ with the ATLAS detector, Eur. Phys. J. C 72 (2012) 2244 [arXiv: 1210.5070] [INSPIRE].

[24] CMS collaboration, Search for heavy neutrinos and $W_{R}$ bosons with right-handed couplings in a left-right symmetric model in pp collisions at $\sqrt{s}=7 \mathrm{TeV}$, Phys. Rev. Lett. 109 (2012) 261802 [arXiv:1210.2402] [INSPIRE].

[25] ATLAS collaboration, ATLAS search for a heavy gauge boson decaying to a charged lepton and a neutrino in pp collisions at $\sqrt{s}=7$ TeV, Eur. Phys. J. C 72 (2012) 2241 [arXiv: 1209.4446] [INSPIRE].

[26] R.N. Mohapatra and G. Senjanović, Neutrino mass and spontaneous parity violation, Phys. Rev. Lett. 44 (1980) 912 [INSPIRE].

[27] R.N. Mohapatra and G. Senjanović, Neutrino masses and mixings in gauge models with spontaneous parity violation, Phys. Rev. D 23 (1981) 165 [INSPIRE].

[28] A. Maiezza, M. Nemevšek, F. Nesti and G. Senjanović, Left-right symmetry at LHC, Phys. Rev. D 82 (2010) 055022 [arXiv: 1005.5160] [INSPIRE].

[29] V. Tello, M. Nemevšek, F. Nesti, G. Senjanović and F. Vissani, Left-right symmetry: from LHC to neutrinoless double beta decay, Phys. Rev. Lett. 106 (2011) 151801 [arXiv:1011.3522] [INSPIRE].

[30] M. Nemevšek, F. Nesti, G. Senjanović and V. Tello, Neutrinoless double beta decay: low left-right symmetry scale?, arXiv:1112.3061 [INSPIRE].

[31] J. Chakrabortty, H.Z. Devi, S. Goswami and S. Patra, Neutrinoless double- $\beta$ decay in TeV scale left-right symmetric models, JHEP 08 (2012) 008 [arXiv:1204.2527] [INSPIRE].

[32] P.S. Bhupal Dev, S. Goswami, M. Mitra and W. Rodejohann, Constraining neutrino mass from neutrinoless double beta decay, Phys. Rev. D 88 (2013) 091301 [arXiv:1305.0056] [INSPIRE]. 
[33] N.T. Shaban and W.J. Stirling, Minimal left-right symmetry and $\mathrm{SO}(10)$ grand unification using LEP coupling constant measurements, Phys. Lett. B 291 (1992) 281 [INSPIRE].

[34] J. Chakrabortty and A. Raychaudhuri, Grand unified theories with dimension-5 interactions: gauge unification and intermediate scales, Phys. Rev. D 81 (2010) 055004 [arXiv:0909.3905] [INSPIRE].

[35] J.F. Gunion, J. Grifols, A. Mendez, B. Kayser and F.I. Olness, Higgs bosons in left-right symmetric models, Phys. Rev. D 40 (1989) 1546 [inSPIRE].

[36] P. Duka, J. Gluza and M. Zralek, Quantization and renormalization of the manifest left-right symmetric model of electroweak interactions, Annals Phys. 280 (2000) 336 [hep-ph/9910279] [INSPIRE].

[37] M. Czakon, J. Gluza and J. Hejczyk, Muon decay to one loop order in the left-right symmetric model, Nucl. Phys. B 642 (2002) 157 [hep-ph/0205303] [INSPIRE].

[38] J. Gluza, On teraelectronvolt Majorana neutrinos, Acta Phys. Polon. B 33 (2002) 1735 [hep-ph/0201002] [INSPIRE].

[39] J. Gluza and M. Zralek, Neutrino production in $e^{+} e^{-}$collisions in a left-right symmetric model, Phys. Rev. D 48 (1993) 5093 [INSPIRE].

[40] R.N. Mohapatra and S. Nussinov, Constraints on decaying right-handed Majorana neutrinos from SN1987a observations, Phys. Rev. D 39 (1989) 1378 [INSPIRE].

[41] M. Czakon, M. Zralek and J. Gluza, Left-right symmetry and heavy particle quantum effects, Nucl. Phys. B 573 (2000) 57 [hep-ph/9906356] [INSPIRE].

[42] A. Belyaev, N.D. Christensen and A. Pukhov, CalcHEP 3.4 for collider physics within and beyond the standard model, Comput. Phys. Commun. 184 (2013) 1729 [arXiv:1207.6082] [INSPIRE].

[43] M.L. Mangano, M. Moretti, F. Piccinini, R. Pittau and A.D. Polosa, ALPGEN, a generator for hard multiparton processes in hadronic collisions, JHEP 07 (2003) 001 [hep-ph/0206293] [INSPIRE].

[44] T. Sjöstrand, S. Mrenna and P.Z. Skands, PYTHIA 6.4 physics and manual, JHEP 05 (2006) 026 [hep-ph/0603175] [INSPIRE].

[45] J. Alwall, M. Herquet, F. Maltoni, O. Mattelaer and T. Stelzer, MadGraph 5: going beyond, JHEP 06 (2011) 128 [arXiv:1106.0522] [INSPIRE].

[46] N.D. Christensen and C. Duhr, FeynRules - Feynman rules made easy, Comput. Phys. Commun. 180 (2009) 1614 [arXiv:0806.4194] [InSPIRE].

[47] C. Degrande et al., UFO - the Universal FeynRules Output, Comput. Phys. Commun. 183 (2012) 1201 [arXiv:1108.2040] [INSPIRE].

[48] D. Guadagnoli and R.N. Mohapatra, TeV scale left right symmetry and flavor changing neutral Higgs effects, Phys. Lett. B 694 (2011) 386 [arXiv: 1008.1074] [INSPIRE].

[49] F. del Aguila, J. de Blas and M. Pérez-Victoria, Effects of new leptons in electroweak precision data, Phys. Rev. D 78 (2008) 013010 [arXiv: 0803.4008] [INSPIRE].

[50] J. Gluza, J. Maalampi, M. Raidal and M. Zralek, Heavy neutrino mixing and single production at linear collider, Phys. Lett. B 407 (1997) 45 [hep-ph/9703215] [INSPIRE]. 
[51] J. Gluza and M. Zralek, On possibility of detecting the $e^{-} e^{-} \rightarrow W^{-} W^{-}$process in the standard model with additional right-handed neutrinos, Phys. Lett. B 362 (1995) 148 [hep-ph/9507269] [INSPIRE].

[52] J. Gluza and M. Zralek, Inverse neutrinoless double beta decay in gauge theories with CP-violation, Phys. Rev. D 52 (1995) 6238 [hep-ph/9502284] [INSPIRE].

[53] W.-Y. Keung and G. Senjanović, Majorana neutrinos and the production of the right-handed charged gauge boson, Phys. Rev. Lett. 50 (1983) 1427 [INSPIRE].

[54] C.-Y. Chen, P.S. Bhupal Dev and R.N. Mohapatra, Probing heavy-light neutrino mixing in left-right seesaw models at the LHC, Phys. Rev. D 88 (2013) 033014 [arXiv:1306.2342] [INSPIRE].

[55] K. Huitu, J. Maalampi, A. Pietila and M. Raidal, Doubly charged Higgs at LHC, Nucl. Phys. B 487 (1997) 27 [hep-ph/9606311] [INSPIRE].

[56] M.L. Swartz, Limits on doubly charged Higgs bosons and lepton flavor violation, Phys. Rev. D 40 (1989) 1521 [InSPIRE].

[57] T.G. Rizzo, Doubly charged Higgs bosons and lepton number violating processes, Phys. Rev. D 25 (1982) 1355 [Addendum ibid. D 27 (1983) 657] [InSPIRE].

[58] J. Maalampi and N. Romanenko, Single production of doubly charged Higgs bosons at hadron colliders, Phys. Lett. B 532 (2002) 202 [hep-ph/0201196] [InSPIRE].

[59] F. del Aguila and J.A. Aguilar-Saavedra, Distinguishing seesaw models at LHC with multi-lepton signals, Nucl. Phys. B 813 (2009) 22 [arXiv:0808.2468] [INSPIRE].

[60] A. Melfo, M. Nemevšek, F. Nesti, G. Senjanović and Y. Zhang, Type II seesaw at LHC: the roadmap, Phys. Rev. D 85 (2012) 055018 [arXiv: 1108.4416] [InSPIRE].

[61] F. del Aguila, M. Chala, A. Santamaria and J. Wudka, Discriminating between lepton number violating scalars using events with four and three charged leptons at the LHC, Phys. Lett. B 725 (2013) 310 [arXiv:1305.3904] [InSPIRE].

[62] K.S. Babu, A. Patra and S.K. Rai, New signals for doubly-charged scalars and fermions at the Large Hadron Collider, Phys. Rev. D 88 (2013) 055006 [arXiv:1306.2066] [INSPIRE].

[63] F. del Aguila and M. Chala, LHC bounds on lepton number violation mediated by doubly and singly-charged scalars, JHEP 03 (2014) 027 [arXiv:1311.1510] [INSPIRE].

[64] J. Pumplin et al., New generation of parton distributions with uncertainties from global QCD analysis, JHEP 07 (2002) 012 [hep-ph/0201195] [INSPIRE].

[65] G. Bambhaniya, J. Chakrabortty, S. Goswami and P. Konar, Generation of neutrino mass from new physics at TeV scale and multi-lepton signatures at the $\mathrm{LHC}$, Phys. Rev. D 88 (2013) 075006 [arXiv: 1305.2795] [INSPIRE].

[66] C.-H. Kom and W.J. Stirling, Charge asymmetry in $W+$ jets production at the LHC, Eur. Phys. J. C 69 (2010) 67 [arXiv:1004.3404] [InSPIRE].

[67] R. Martinez, M.A. Perez and J.J. Toscano, The two photon decay width of the Higgs boson in left-right symmetric theories, Phys. Rev. D 40 (1989) 1722 [INSPIRE].

[68] I. Picek and B. Radovcic, Enhancement of $h \rightarrow \gamma \gamma$ by seesaw-motivated exotic scalars, Phys. Lett. B 719 (2013) 404 [arXiv:1210.6449] [InSPIRE].

[69] M. Carena, I. Low and C.E.M. Wagner, Implications of a modified Higgs to diphoton decay width, JHEP 08 (2012) 060 [arXiv:1206.1082] [INSPIRE]. 
[70] ATLAS collaboration, Measurements of the properties of the Higgs-like boson in the two photon decay channel with the ATLAS detector using $25 \mathrm{fb}^{-1}$ of proton-proton collision data, ATLAS-CONF-2013-012, ATLAS-COM-CONF-2013-015 (2013).

[71] CMS collaboration, Updated measurements of the Higgs boson at $125 \mathrm{GeV}$ in the two photon decay channel, CMS-PAS-HIG-13-001 (2013).

[72] P.S. Bhupal Dev, D.K. Ghosh, N. Okada and I. Saha, $125 \mathrm{GeV}$ Higgs boson and the type-II seesaw model, JHEP 03 (2013) 150 [Erratum ibid. 05 (2013) 049] [arXiv:1301.3453] [INSPIRE].

[73] A.G. Akeroyd and S. Moretti, Enhancement of $H \rightarrow \gamma \gamma$ from doubly charged scalars in the Higgs triplet model, Phys. Rev. D 86 (2012) 035015 [arXiv:1206. 0535] [INSPIRE].

[74] J. Gluza and M. Zralek, Higgs boson contributions to neutrino production in $e^{-} e^{+}$collisions in a left-right symmetric model, Phys. Rev. D 51 (1995) 4695 [hep-ph/9409225] [INSPIRE].

[75] G. Ecker, W. Grimus and H. Neufeld, Higgs induced flavor changing neutral interactions in $\mathrm{SU}(2)_{L} \times \mathrm{SU}(2)_{R} \times \mathrm{U}(1)$, Phys. Lett. B 127 (1983) 365 [Erratum ibid. B 132 (1983) 467] [INSPIRE].

[76] R.N. Mohapatra, G. Senjanović and M.D. Tran, Strangeness changing processes and the limit on the right-handed gauge boson mass, Phys. Rev. D 28 (1983) 546 [INSPIRE].

[77] M.E. Pospelov, FCNC in left-right symmetric theories and constraints on the right-handed scale, Phys. Rev. D 56 (1997) 259 [hep-ph/9611422] [INSPIRE]. 